\title{
Wavelet analysis on transient behaviour of tidal amplitude fluctuations observed by meteor radar in the lower thermosphere above Bulgaria
}

\author{
D. Pancheva ${ }^{1,2}$, P. Mukhtarov ${ }^{1}$ \\ ${ }^{1}$ Geophysical Institute, Bulgarian Academy of Sciences “Acad. G. Bonchev str.”, bil.3, 1113 Sofia, Bulgaria \\ 2 Physics Department, The University of Wales, Aberystwyth, SY23 3BZ, UK, e-mail: ddp@aber.ac.uk
}

Received: 16 December 1998 / Revised: 19 November 1999 / Accepted: 30 November 1999

\begin{abstract}
On the basis of bispectral analysis applied to the hourly data set of neutral wind measured by meteor radar in the MLT region above Bulgaria it was demonstrated that nonlinear processes are frequently and regularly acting in the mesopause region. They contribute significantly to the short-term tidal variability and are apparently responsible for the observed complicated behavior of the tidal characteristics. A Morlet wavelet transform is proposed as a technique for studying nonstationary signals. By simulated data it was revealed that the Morlet wavelet transform is especially convenient for analyzing signals with: (1) a wide range of dominant frequencies which are localized in different time intervals; (2) amplitude and frequency modulated spectral components, and (3) singular, wavelike events, observed in the neutral wind of the MLT region and connected mainly with large-scale disturbances propagated from below. By applying a Morlet wavelet transform to the hourly values of the amplitudes of diurnal and semidiurnal tides the basic oscillations with periods of planetary waves (1.5-20 days), as well as their development in time, are obtained. A cross-wavelet analysis is used to clarify the relation between the tidal and mean neutral wind variability. The results of bispectral analysis indicate which planetary waves participated in the nonlinear coupling with the atmospheric tides, while the results of cross-wavelet analysis outline their time intervals if these interactions are local.
\end{abstract}

Key words: Meteorology and atmospheric dynamics (middle atmosphere dynamics; waves and tides) - Radio science (nonlinear phenomena)

\section{Introduction}

Long time series of tidal observations in the mesosphere/ lower thermosphere (MLT) region are characterized as being variable at different time scales, from short-term variations (a few days) to long-term changes (several months or years, including the seasonal cycle). Long term variations of the atmospheric tides can have mainly two causes (Vial, 1992): changes in the tidal forcing and/ or changes in the structure of the middle atmosphere through which the tides propagate. The solar cycle is presumed to influence the solar tides, particularly the semidiurnal tide, which is generated in the stratosphere by ozone absorption of UV solar radiation. Most of the long-term continuous observations showed a negative correlation for the amplitude of the semidiurnal tide (Sprenger and Schminder, 1969; Dartt et al., 1983; Greisiger et al., 1987; Namboothiri et al., 1993), but the statistical significance of these results was generally low. Jacobi et al. (1997) found no solar cycle dependence of the semidiurnal amplitude and pointed out that this may be due to the observed oscillation at very long time scales that is obviously of non-solar origin. Bremer et al. (1997) found a long-term decrease of the semidiurnal tide, which is in correspondence with ozone depletion in the stratosphere.

One of the inherent features of the stratospheric circulation, which should influence the tidal characteristics, is the Quasi-Biennial Oscillation (QBO) in the equatorial region. Even though the theoretical results (Hagan et al., 1992) indicated that the semidiurnal tide induced in the middle atmosphere and reaching the thermosphere is indeed modulated by the stratosphere QBO, there is no study demonstrating any possible QBO effect on the solar tides measured in the MLT region. However, there are investigations presenting evidence that the interannual variability of planetary waves, observed in the MLT region, shows a dependence on the QBO. Espy et al. (1997) and Jacobi (1998) have shown that QBO influences the quasi 16-day wave activity in 
the summer. They suggested that during the easterly phase of the QBO the wave propagation is blocked by the equatorial stratospheric easterly winds, so that the planetary wave activity in the polar mesosphere is strongly reduced.

The latitudinal and vertical behavior of the atmospheric tides as a function of season is quite well established (Avery et al., 1989; Manson et al., 1989; Vincent et al., 1989). Modeling studies (Vial, 1986; Forbes and Hagan, 1988; Forbes and Vial, 1989; Hagan et al., 1995) have made progress in predicting some aspects of tidal seasonal variability.

Day-to-day tidal variability is more difficult to be explained and is probably a result of several different mechanisms. Recent studies have revealed that strong wave-wave nonlinear interaction processes of gravity waves, tides and planetary waves in the MLT region play an important role in the dynamics of the middle atmosphere and certainly contribute significantly to the short-term tidal variability (Fritts and Vincent, 1987; Manson and Meek, 1990; Huuskonen et al., 1991; Cevolani and Kingsley, 1992; Rüster, 1992, 1994; Ma and Schlegel, 1993; Pancheva and Mukhtarov, 1994; Mitchell et al., 1996; Kamalabadi et al., 1997). The theoretical studies of nonlinear wave-wave interaction (Teitelbaum et al., 1989; Teitelbaum and Vial, 1991) proposed that two primary waves can interact through the nonlinear advection terms of the momentum equation and produce, among others, two secondary waves whose frequencies, wave numbers and phases are the sum and difference of the frequencies, wave number and phases of the primary waves. The secondary waves may beat with the primary waves and modulate the amplitude of the shorter period one with the period of the other. The occurrence of a nonlinear coupling between planetary waves and tides results in a modulation of the tidal amplitudes at planetary wave periods. The process of nonlinear interaction can take place in the MLT region, in which case it is termed a local interaction, and then the planetary wave itself has to be observed there. However, it has been proposed (Carter and Balsley, 1982; Mitchell et al., 1996) that nonlinear interaction may have taken place at other heights and, as the propagation conditions could be different for the modulated wave and the planetary wave, the latter might not propagate to the MLT region. This process may transmit the influence of planetary waves to greater heights through the modulation of the ascending tides or gravity waves.

The nonlinear interaction between semidiurnal and diurnal tides is responsible not only for the variability of the terdiurnal tide, but for the diurnal tide also (Teitelbaum et al., 1989). A secondary diurnal tide is generated and the superposition of the primary and secondary diurnal tides can be highly variable even if the primary tides exhibit small changes.

The nonlinear interaction between different planetary waves is also possible. Recently, Jacobi et al. (1998) showed that nonlinear interaction between the quasi2-day wave and long-period planetary waves (mainly 10 and 16-day waves) is a regular process in the upper mesosphere over Central Europe. Analogous result was obtained by Pancheva et al. (1999) investigating neutral wind observed in the MLT region above Sheffield (UK) during 1992 and 1993. This process of planetary waveplanetary wave coupling could influence the tidal variability also through the nonlinear interaction between tides and planetary waves. Consequently, an essential part of a short-term tidal variability is related to the nonlinear interaction processes, which presumably are a common feature of the dynamics of the MLT region.

The aim of the present work is to describe the variability of the atmospheric tides, in the periodic range 1.5-20 days, observed by meteor radar in the MLT region above Bulgaria during the interval January, 1991-June, 1992, using wavelet transform as a technique for studying nonstationary signals. Since this technique regards a fluctuating data series as a superposition of localized similar waves, it should describe appropriately the distribution of localized and transient features, such as atmospheric waves in the middle atmosphere.

\section{Measurements and data analysis}

A meteor radar has operated regularly in Bulgaria (Yambol, $42.5^{\circ} \mathrm{N}, 26.6^{\circ} \mathrm{E}$ ) since the end of 1989 and the measurements have been processed and analyzed in the Geophysical Institute, Sofia since the end of 1990. The meteor radar provides integrated measurements of a weighted wind profile about $95 \mathrm{~km}$ (Lysenko et al., 1988). The measurements were obtained by sounding simultaneously in four geographical directions, however disturbances in two of them (east and north) during most of the day-time after April 1991, resulted in only the measurements of the western and southern antennae being used in processing and analyzing the data. This fact is a reason why the level of noise for the measurements after April, 1991, is higher than the previous ones; the north and south measurements were averaged together to produce a single data set corresponding to the meridional component of the neutral wind and similarly, the east and west measurements produce a single zonal component. The measurements were averaged to produce one value per hour in the zonal and meridional directions, so the minimum identifiable period is $2 \mathrm{~h}$ (Nyquist period). The main features of the monthly mean dynamical regime in the MLT region above Bulgaria were described by Pancheva and Mukhtarov (1994). The characteristics of the observed quasi-2-day variations and the influence of the winter stratospheric warming on the tidal variability were also briefly considered there. An empirical seasonal model of the tidal wind was created by Pancheva and Mukhtarov (1996) using daily values of tidal characteristics. In addition to the well known annual and semiannual waves, the influence of shorter seasonal components (2-month component, for example) on some tidal characteristics was observed.

Studies by Williams and Avery (1992), Cevolani and Kingsley (1992) and Vincent (1993) indicated oscillations with periods ranging from 1.5 to 20 days in the MLT 
region to be consistent with those found in the lower altitudes. The same period interval of tidal variability is under investigation in this work also. To obtain a general view of the main spectral components observed in the amplitudes of diurnal and semidiurnal tides, we calculate the amplitude spectra for zonal (Fig. 1) and meridional (Fig. 2) wind components derived by the method of correloperiodogram analysis (Kopecky and Kuklin, 1971; description of this method can be found also in Apostolov et al., 1995) and applied to the daily values of tidal wind. To compare the oscillations in the tidal amplitudes with periods of planetary waves to those in the neutral wind the amplitude spectra of the prevailing zonal (Fig. 1c) and meridional (Fig. 2c) wind are calculated also. The horizontal dashed lines in all spectra represent the $95 \%$ confidence level. In general, the spectral picture is complicated; several period bands can be distinguished where the spectral peaks have higher than $95 \%$ confidence level. The periodic bands for the tidal amplitudes in zonal wind are 4-5 (only for semidiurnal tide), 7, 9-10 and around 16-17 days. There is also a 19-day oscillation in the amplitudes of semidiurnal tide (Fig. 1a) and an 11-12-day oscillation in the amplitudes of diurnal tide (Fig. 1b). Similar spectral components can be seen in the amplitude spectrum of the prevailing zonal wind (Fig. 1c), however the very well-expressed 12-13-day oscillation in the prevailing zonal wind cannot be seen in the spectra of tidal amplitudes. The well-outlined periodic bands for the tidal amplitudes in the meridional wind are at $\sim 2.5$, 4-5, a broad band around 8-10 and 16-17 days for diurnal tide (Fig. 2b) and a very well-expressed peak around 14 days for the semidiurnal tide (Fig. 2a). Again similar spectral components can be distinguished in the amplitude spectrum of the prevailing meridional wind (Fig. 2c). Some of the periodic bands (for example 8-10, or 16-18 days) are quite broad and this is connected with the temporal changes of the prevailing period.

In order to study the complicated behavior of the amplitude variations in tidal velocities the time series of hourly values of tidal amplitudes are analyzed. Pancheva and Mukhtarov (1994) found that the main tidal components describing the dynamics of the MLT region above Bulgaria are semidiurnal, diurnal, terdiurnal and quarterdiurnal tides. Least-squares fits were applied to 24-hour segments of wind data to calculate the prevailing wind and amplitudes and phases of the above mentioned atmospheric tides. The derived characteristics are assigned to the center of the segment. No fit was attempted if less than $16 \mathrm{~h}$ of data were present in any 24-h segment. In order to obtain the following hourly values of prevailing wind and tidal characteristics the 24-h segment was incremented in one-hour steps. In this way the hourly values of tidal characteristics and prevailing wind were produced. The time interval under consideration, January 1991-June 1992, was divided into two subintervals: 01 January-23 October 1991, and 21 November 1991-16 June 1992, as the data for the interval 24 October-20 November 1991 have many gaps. Mukhtarov and Pancheva (1993) describe this procedure in detail. An analogous approach was used by Mitchell
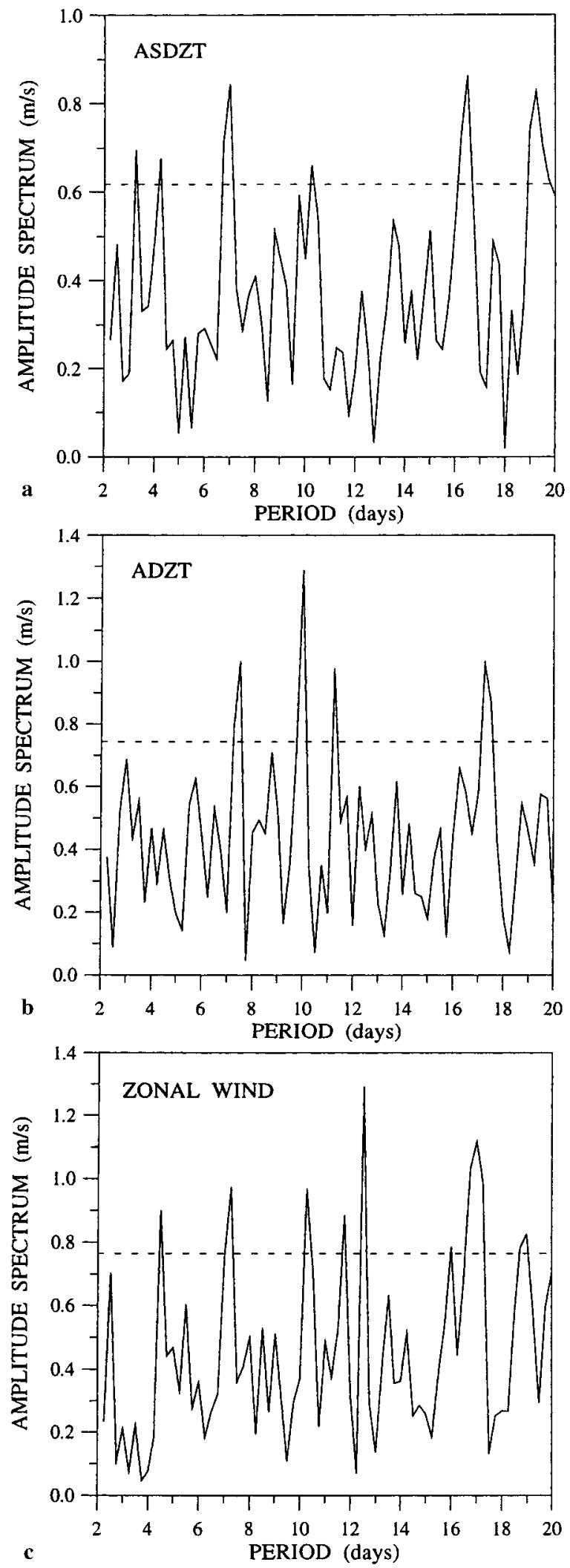

Fig. 1a-c. The amplitude spectra for: a the amplitudes of semidiurnal tide in the zonal wind $(A S D Z T)$; $\mathbf{b}$ the amplitudes of diurnal tide in the zonal wind $(A D Z T)$; $\mathbf{c}$ the prevailing zonal wind, obtained by the method of correloperiodogram analysis, applied to the daily values of tidal wind. The horizontal dashed lines in all spectra represent the $95 \%$ confidence level 

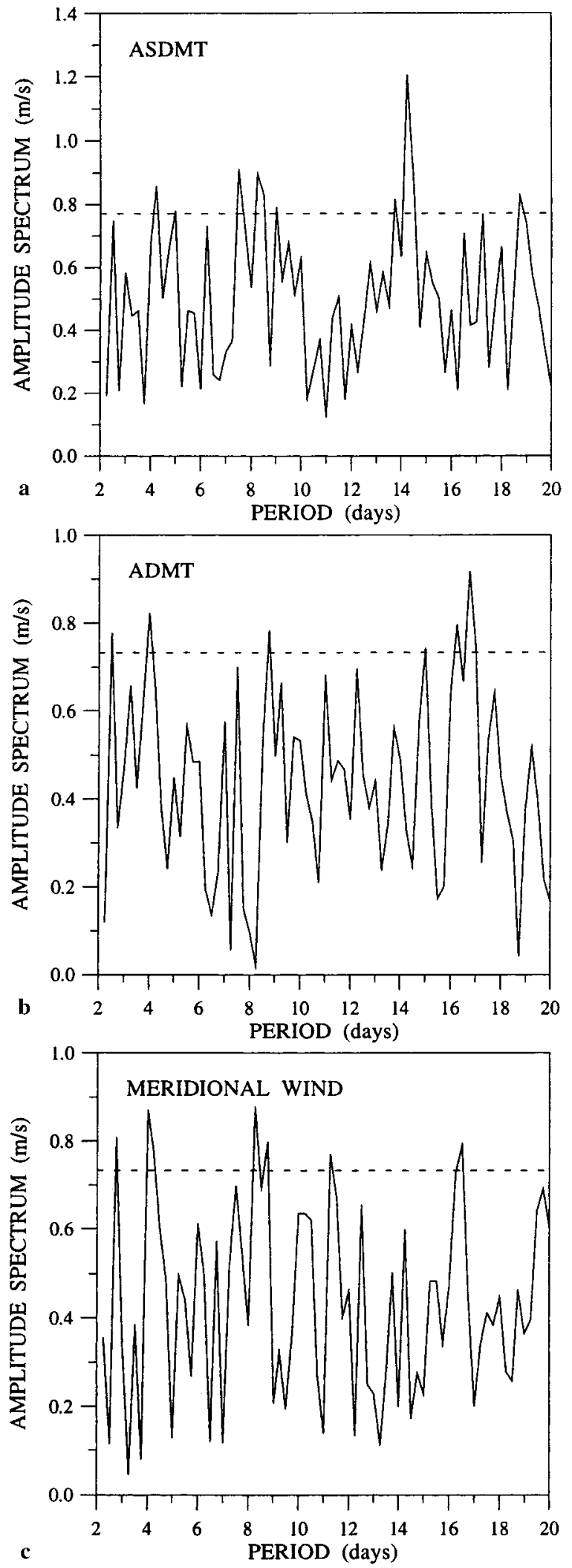

Fig. 2a-c. The same as Fig. 1, but for: a the amplitudes of the semidiurnal tide in the meridional wind $(A S D M T)$; $\mathbf{b}$ the amplitudes of the diurnal tide in the meridional wind $(A D M T)$; $\mathbf{c}$ the prevailing meridional wind et al. (1996) investigating the variability of semidiurnal tides, and also by Pancheva and Mukhtarov (1998) studying the response of the MLT region dynamics to major geomagnetic storms.

The complicated behavior of the tidal variability is probably related to the nonlinear processes which are frequently and regularly acting in the MLT region over Bulgaria during the interval under consideration. To clarify this topic we consider in detail some fluctuations of the tidal amplitudes whose periods correspond to the spectral peaks in Figs. 1 and 2 with confidence level higher than $95 \%$. Figure 3 a shows a 2.5 -day wave in the amplitudes of the diurnal meridional tide (the respective 2.5-day peak is marked on Fig. 2b), observed during January-March 1991. These oscillations are separated by a band-pass filter with a linear phase, described by Luzov et al. (1965), centered at the required peak and which suppressed low and high frequency variations (filter characteristics can be found in Pancheva et al., 1994). The quasi-2-day wave in the diurnal amplitude is a strong wave phenomenon with amplitudes close to 10 $12 \mathrm{~m} / \mathrm{s}$. The analogous wave can also be observed in the meridional neutral wind (Fig. 2c) and this is an example of the quasi-2-day wave in the winter MLT region. To prove that the quasi-2-day wave in the amplitudes of the diurnal tide is a result of a nonlinear interaction between the diurnal tide and the quasi-2-day wave in the meridional wind, the bispectral analysis technique is applied to the time series of hourly wind measurements. The bispectrum measures the statistical dependence between all sets of three frequencies where two of them sum to yield the third. Various bispectrum algorithms and their properties are outlined in Kim and Powers (1979) and Nikias and Raghuveer (1987). The conventional "Fourier type" methods for bispectrum estimation are usually used in investigating the nonlinear interaction between waves in the MLT region (Rüster, 1992, 1994; Clark and Bergin, 1997; Kamalabadi et al., 1997; Beard et al., 1999; Pancheva, 1999). In the present work the magnitude squared bispectrum is calculated from the hourly values of the neutral wind. The computational procedure is based on the segmentation of data (overlapping segments). After removing the mean value the complex Fourier components on each of the windowed segment, treated as independent components of an ensemble average, were calculated. The bispectrum was estimated by averaging the complex products of all triplets of complex Fourier components at frequencies where two of them sum to yield the third. As the bispectrum requires an ensemble of independent complex Fourier transforms for its computation, it has considerably more stringent stationarity requirements than a power spectrum of comparable frequency resolution. This could be a problem when we use long time segments; this will improve frequency resolution, but may introduce potential nonstationarities. So, the data have to be stationary enough to allow a bispectral computation, which is a useful tool for discriminating phase-coupling components from those that are not.

The contour plot of the calculated magnitude squared bispectrum for the meridional wind during 

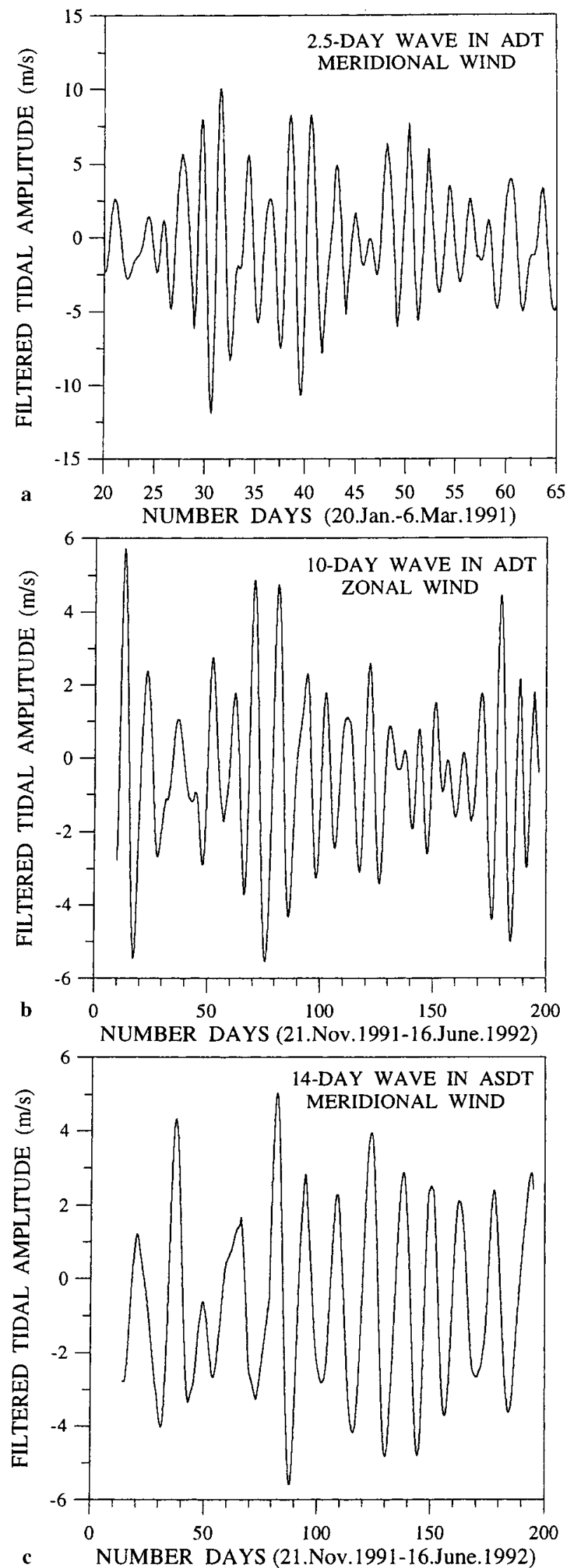

01 January-23 October 1991 in the periodic interval $0.25-20$ days is shown on Fig. 4a. The non-zero points in this plane indicate the quadratic nonlinear interaction between waves with periods corresponding to the respective coordinate values of these points. As we use finite-length time series, even a process with truly independent Fourier components will have a nonzero bispectrum. Significance levels of zero bispectrum must be known to determine if data are statistically consistent with a linear, random phase process. The variance of bispectral estimates must be approximately computed in order to use this method as a test for nonlinear effects in stationary time series. A desirable parameter, proposed by Haubrich (1965), is the $95 \%$ confidence limits for the bicoherence when the true value of the bispectrum is zero. He has shown that if the studied process is stationary and normally distributed the square of bicoherence has to be less than about $6 / v$, where $v$ is the degree of freedom (see also Elgar and Guza, 1988). The empirically derived value appears to be about $4 / v$ for the 95\% confidence limits (Haubrich, 1965). Using the theoretical result we can roughly estimate the bispectral level for the process which is to be distinguished from a Gaussian process with $95 \%$ confidence. In Lii et al. (1976) and Lii and Helland (1981) a method for direct estimation of the bispectral variance is given and it was demonstrated that estimated and theoretical asymptotic variance of the bispectrum are comparable. Following strictly this mentioned method for direct estimation of the bispectral variance and having in mind the expressions for bicoherence, given by Nikias and Raghuveer (1987) and for asymptotic bispectral variance, first derived by Brillinger and Rosenblatt (1967), an approximate estimate of the bispectral level for a non Gaussian process with $95 \%$ confidence can be obtained: $\left|B\left(\omega_{k}, \omega_{l}\right)\right|^{2} \geq C(v, m, L)\left[S D\left(\omega_{k}, \omega_{l}\right)\right]^{2}$, where $S D\left(\omega_{k}, \omega_{l}\right)$ is the standard deviation of the bispectral variance for the frequencies $\omega_{k}$ and $\omega_{l}$, calculated by the method described in Lii et al. (1976) and Lii and Helland (1981) and $C$ is a parameter depending on the degrees of freedom $v$, the number of segments $m$ and the sample size $L$. In our case the bispectra are calculated by ensemble averaged, producing bispectral estimates with $2 m$ degrees of freedom and then the approximate value of $C$ is 2 (for the empirical condition the value of $C$ is about 1.4). For all frequency pairs, participating in the non-linear interactions under consideration the standard deviations of the bispectral variance were calculated and it is indicated that the condition is fulfilled.

Figure $4 \mathrm{a}$ clearly outlined the planetary waves nonlinearly coupling with diurnal and semidiurnal tides. The 2.5 -day wave is among the planetary waves interacting with the diurnal tide (the magnitude squared bispectrum in this case is $8.62 \mathrm{E}+06$ and the calculated $(S D)^{2}$ for the

Fig. 3a-c. The filtered tidal amplitude data for: a 2.5-day wave in the amplitudes of the diurnal meridional tide in January-March 1991; b 10-day wave in the amplitudes of diurnal zonal tide between 21 November 1991-16 June 1992, and c 14-day wave in the amplitudes of semidiurnal meridional tide 21 November 1991-16 June 1992 

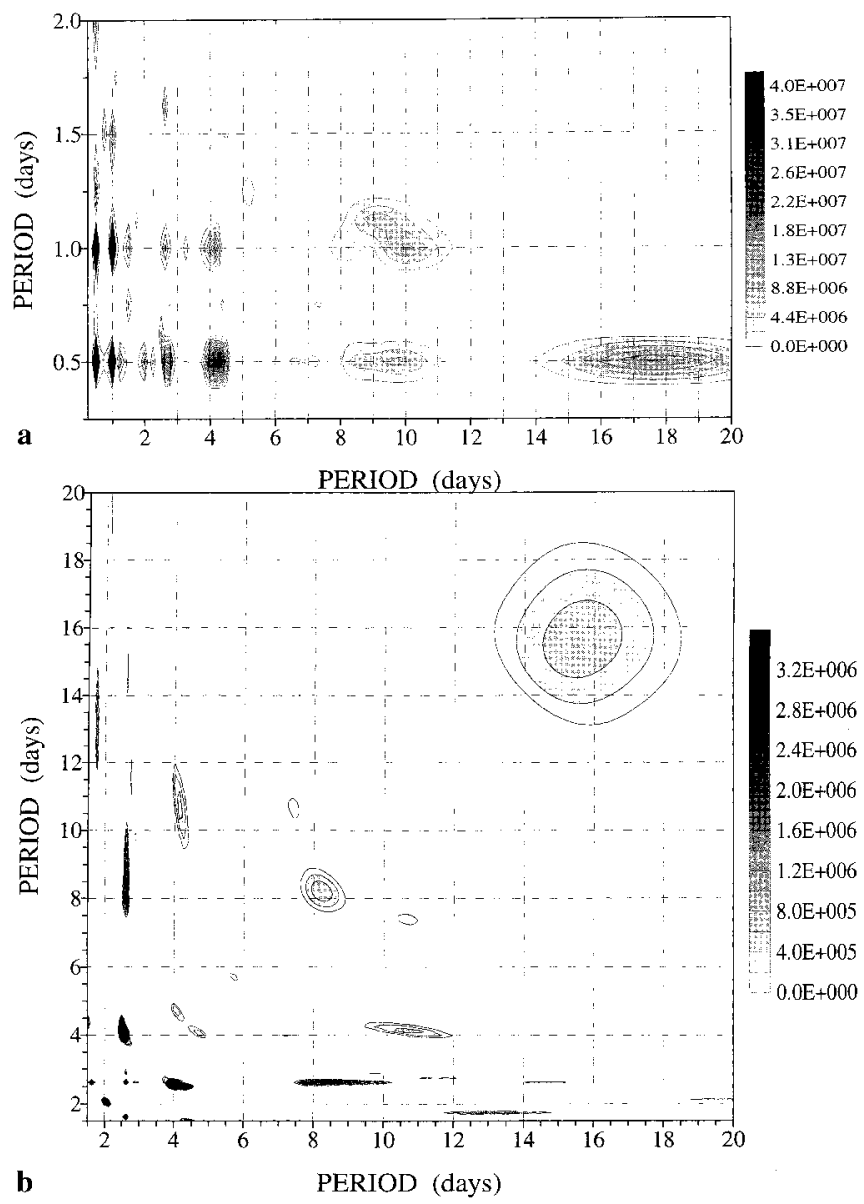

Fig. 4a, b. The contour plot of the magnitude squared bispectrum for the meridional wind during 01 January-23 October 1991 in the periodic interval: a $0.25-20$ days, and b 1.5-20 days

diurnal tide and 2.5-day wave is $2.54 \mathrm{E}+06$ ), so the welldeveloped 2.5-day wave in the amplitudes of the diurnal tide, shown on Fig. 3a, is really a result of the nonlinear coupling process. The non-linear tidal/quasi-2-day interaction is investigated also by Walterscheid and Vincent (1996), who suggested that the phase-locked largeamplitude summertime 2-day wave in the Southern Hemisphere is a nearly resonant wave forced parametrically by the zonally symmetric diurnal tides. They proposed also that the 2-day wave can be generated by a set of non-linear mutually reinforcing interactions. The process involves the self-excitation of the 2-day wave by an interaction with a diurnal zonal wave number 6 tide, itself generated nonlinearly in a two-step process initiated by the interaction of the 2-day wave and the migrating tides. Recent numerical simulations have been conducted using the NCAR TIME-GCM and evidence for nonlinear interactions between the quasi-2-day wave and migrating tides is presented (Palo et al., 1998, 1999). Three wave packets with strongly modulated amplitudes can be clearly distinguished on Fig. 3a. This amplitude modulation of the 2.5-day wave in the diurnal meridional tide can be also a consequence of a nonlinear interaction process, but between different planetary waves present in the meridional wind. The contour plot of the calculated magnitude squared bispectrum for the meridional wind in the period interval 1.5-20 days is displayed in Fig. 4b. The non-zero points in this plane indicate the nonlinear interactions between different planetary waves in the period range mentioned. The strongest interactions are those between 2.5- and 4-day waves (in this case the magnitude squared bispectrum is more than an order of magnitude larger than the square of $S D$ ), as well as between 2.5- and 8-10-day waves (the magnitude squared bispectrum is $1.67 \mathrm{E}+06$ and the square of $S D$ is $4.2 \mathrm{E}+05$ ). There are also coupling of the 2.5-day wave with $\sim 11-12$-day waves (in this case only an empirical $95 \%$ confidence limit is fulfilled). The obtained result supports the idea that amplitude modulation of the 2.5-day wave in the amplitudes of diurnal tide is a consequence of the quadratic nonlinear coupling between 2.5- and longer 8-10-, or 11-12-day waves.

Figure $3 \mathrm{~b}$ shows a 10-day wave present in the amplitudes of the zonal diurnal tide (the respective peak is marked on Fig. 1b), while Fig. 3c shows a 14-day wave in the amplitudes of a meridional semidiurnal tide (the respective peak is visible on Fig. 2a). Both wave phenomena are observed during the second subinterval (21 November 1991-16 June 1992) and indicate welldeveloped planetary waves with amplitudes reaching 5-6 m/s. To prove that the observed periodic tidal variability is a result of nonlinear interaction between tides and planetary waves we use bispectral analysis again. The contour plot of Fig. 5a describes the nonlinear coupling between tides and planetary waves in the zonal wind, while Fig. $5 \mathrm{~b}$ shows the same in the meridional wind, both during the second subinterval. The nonlinear interaction between the diurnal tide and the 10-day planetary wave in the zonal wind is clearly visible on Fig. 5a (the magnitude squared bispectrum is $2.97 \mathrm{E}+06$ and the squared $S D$ is $4.59 \mathrm{E}+05$ ), while the coupling between the semidiurnal tide and 14-day wave in the meridional wind - on Fig. 5b (the magnitude squared bispectrum is $3.12 \mathrm{E}+07$ and the squared $S D$ is $6.28 \mathrm{E}+06$ ). There are also strong nonlinear interactions of the semidiurnal tide with 7- and 10-day planetary waves in the zonal wind, shown on Fig. 5a (the magnitude squared bispectrum are respectively $4.32 \mathrm{E}+06$ and $4.47 \mathrm{E}+06$ and the respective squared $S D$ are $7.46 \mathrm{E}+05$ and $7.08 \mathrm{E}+05$ ). So, the respective spectral peaks at 7 and 10 day, indicated on Fig. 1a, are also a result of nonlinear interaction processes. The amplitude modulation is clearly present on Fig. 3b, c, so the 10- and 14-day waves in the tidal amplitudes probably participated in nonlinear coupling with longer-period fluctuations, whose main periods belong to the seasonal or intraseasonal components.

These results strongly support the nonlinear character of the tidal variability observed by meteor radar in the MLT region above Bulgaria.

\section{Wavelet transform}

Fourier analysis has been widely used to interpret the observational results for different atmospheric waves in 

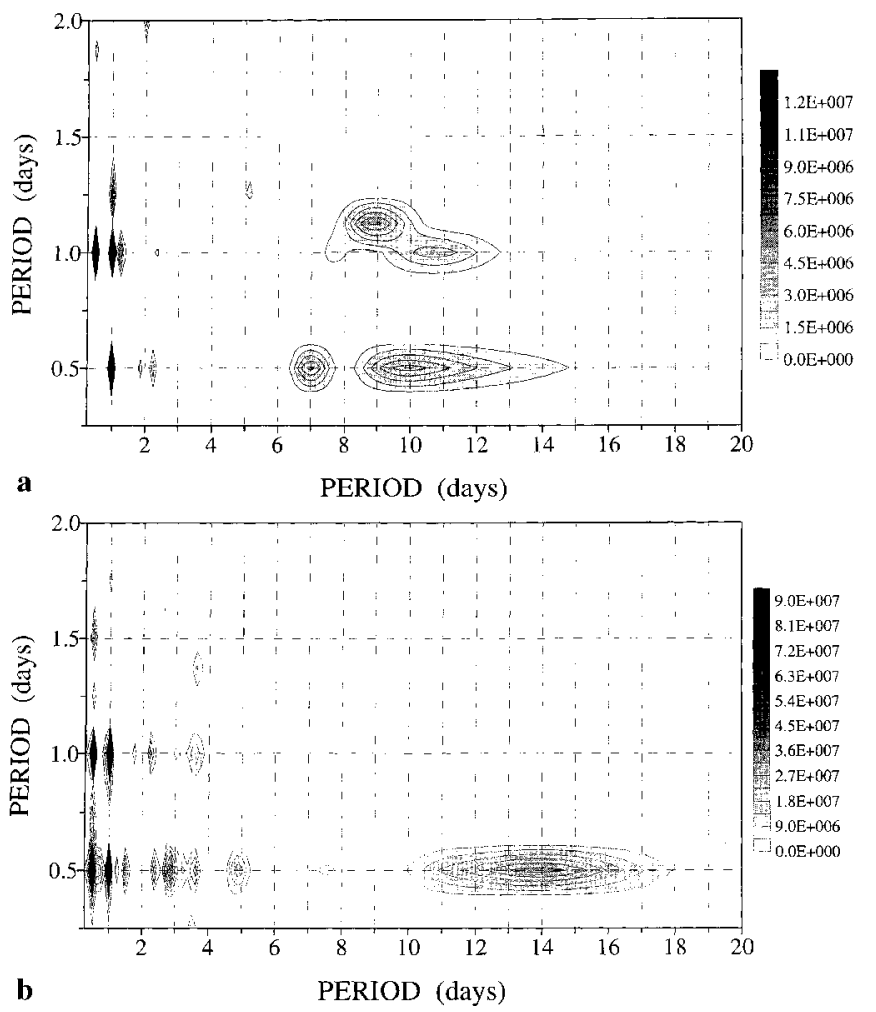

Fig. 5a, b. The contour plot of the magnitude squared bispectrum in the periodic interval $0.25-20$ days for: a the zonal wind, and b the meridional wind, during the interval 21 November 1991-16 June 1992

the middle atmosphere, assuming that there are monochromatic waves that are continued infinitely in time and space. However, continuous spectral analysis and monochromatic analysis are both partly successful in observational studies (Manson et al., 1982; Fritts, 1989; Manson and Meek, 1990; Huuskonen et al., 1991; Vial, 1992; Beard et al., 1997). When a data series includes different kinds of localized or intermittent events, or some frequencies demonstrate complicated temporal behavior, the Fourier spectrum is usually a complex mixture, which is difficult to interpret. Fourier transform, for example, cannot distinguish between a signal representing the sum of two components with different frequencies, and a signal, consisting of the same components included consecutively. One of the reasons for this difficulty is that the Fourier spectrum (the squared modulus of the Fourier coefficients), lacks the information of the position of the events which is carried in the phases of the Fourier coefficients. An obvious method for dealing with short-time or nonstationary signals is the Fourier transform with a sliding time segment (short-time Fourier transform). The location of the sliding segment adds a time dimension and one gets a time-varying frequency analysis. Unfortunately, as the trigonometric functions are defined to infinity, the short-time Fourier transform also spreads information about the localized features over all frequencies making it impossible to confidently localize these features. If a window function is used and if it is the Gaussian function, one has a Gabor wavelet, named after Denis Gabor who used it in the analysis of radar signals in the late 1940s (Gabor, 1946). A disadvantage of the Gabor wavelet is that the window function is fixed and therefore as the frequency is high there are more cycles included inside the window. Hence, the Gabor wavelet does not treat all frequency components of the signal in the same way.

The wavelet transform is an alternative to the windowed Fourier transform and can be used to analyze time series that contain nonstationary power at many different frequencies. By decomposing the time series into time-frequency space, one is able to determine both the dominant modes of variability and how those modes vary in time. The essence of the wavelet transform is based on the idea of decomposing the signal into building blocks of constant "shape" but different size (Daubecheies, 1990). It uses analyzing functions, called wavelets, which are localized in space. The limited spatial support of the wavelets is important because then the behavior of the signal at infinity does not play any role. The spectral resolution is achieved by selection of the wavelet size (or by dilating or contracting of the chosen wavelet) and the temporal resolution follows from the location of the wavelet relative to the signal. As contracted (or dilated) version of the basic wavelet is adapted to the frequency range, then the higher that range, the narrower the wavelet function is. Note that for higher frequencies the time resolution improves and this enables the wavelet transform to perform better than the windowed Fourier transform signals which typically have short-lived highfrequency components superposed on longer-lived lower frequency parts.

\subsection{The continuous wavelet transform}

The only constraint on a function $h(t)$, real- or complexvalued, in order to be a wavelet, is the admissibility condition and that it should also be well-localized in both physical and Fourier space (Farge, 1992; Meyer, 1992). The wavelet transform of a function $y(t)$ is defined as:

$W(a, b)=a^{-1 / 2} \int_{-\infty}^{\infty} y(t) h^{*}\left(\frac{t-b}{a}\right) \mathrm{d} t$

where the $(*)$ indicates the complex conjugate and the variable $a$ is the scale dilation parameter and $b$ the translation parameter. The real- or complex-valued function $h(t)$ is called a mother (or analyzing) wavelet. The admissibility condition can be expressed:

$C_{h}=\int_{-\infty}^{\infty}|H(\omega)|^{2} \frac{\mathrm{d} \omega}{|\omega|}<\infty$

where $H(\omega)$ is the Fourier transform of $h(t)$, 
$H(\omega)=\int_{-\infty}^{\infty} h(t) \exp (-i \omega t) \mathrm{d} t$

If $h(t)$ is integrable then Eq. (2) implies that it has a mean of zero: $H(0)=\int_{-\infty}^{\infty} h(t) \mathrm{d} t=0$. The admissibility condition ensures that the inverse transform and Parseval formula are applicable. The factor $a^{-1 / 2}$ in the right hand part of Eq. (1) keeps the energy at each scale independent of $a$. The wavelet transform coefficient $W(a, b)$ can be interpreted as being the relative contribution of fluctuations at scale $a$ to the signal at position $b$.

An important property of the wavelet transform is conservation of energy (Parseval formula):

$$
\int_{-\infty}^{\infty}|y(t)|^{2} \mathrm{~d} t=C_{h}^{-1} \int_{0}^{\infty} \frac{\mathrm{d} a}{a^{2}} \int_{-\infty}^{\infty}|W(a, b)|^{2} \mathrm{~d} b
$$

The integrand on the right can be considered to be the energy density at a given location in wavelet space. When it is integrated over $b$ the wavelet energy spectrum is obtained:

$P(a)=C_{h}^{-1} a^{-2} \int_{-\infty}^{\infty}|W(a, b)|^{2} \mathrm{~d} b$

A Morlet wavelet is used in the present work. It is obtained by taking a complex sine wave and localizing it with a Gaussian (bell-shaped) envelope.

$g(\eta)=\pi^{-1 / 4} \exp \left(i \omega_{0} \eta\right) \exp \left(-\eta^{2} / 2\right)$

where $\eta$ is nondimensional "time" parameter and $\omega_{0}$ is the nondimensional frequency, which gives the number of oscillations within the wavelet itself. Here it is taken to be 6 to satisfy the admissibility condition (Farge, 1992). For large $\omega_{0}$ the frequency resolution improves; though at the expense of decreased time resolution. We choose the Morlet wavelet as it is simple and looks like a modulated wave "packet", which we observed frequently in the tidal amplitudes data. In our case of continuous Morlet wavelet transform we denote: $T=a T_{0}$, where $T_{0}=2 \pi / \omega_{0}=$ const, as in our case $\omega_{0}=6$. Bearing in mind that our measurements have time step of one hour (due to aliasing the maximum frequency detected is 0.5 hour $^{-1}$ ) and changing symbol: $b \rightarrow t_{k}$ we obtained:

$$
\begin{aligned}
W\left(T, t_{k}\right)= & \left(\frac{T_{0}}{T}\right)^{1 / 2} \sum_{i=0}^{N-1} y\left(t_{i}\right) \exp \left[-j \frac{2 \pi}{T}\left(t_{i}-t_{k}\right)\right] \\
& \times \exp \left[-\frac{T_{0}^{2}}{2}\left(\frac{t_{i}-t_{k}}{T}\right)^{2}\right]
\end{aligned}
$$

In the case of an one-dimensional signal, the continuous wavelet transform generates two-dimensional (period/or scale and time) wavelet space, and therefore it is a redundant representation of the signal. The Morlet wavelet transform will have a real and imaginary part, or can be represented as magnitude and phase. The magnitude of the transform is related to the local energy, while the phase completes the representation.
Just as in the case of a Fourier transform, both real and imaginary parts must be known in order to calculate an inverse transform and reconstruct the signal.

\subsection{Wavelet applications on synthetic time series}

To demonstrate the abilities of the chosen Morlet wavelet function some synthetic signals are analyzed. Figure 6a shows a time series of a length $2880 \mathrm{~h}$, as the signal is formed by the following waves:
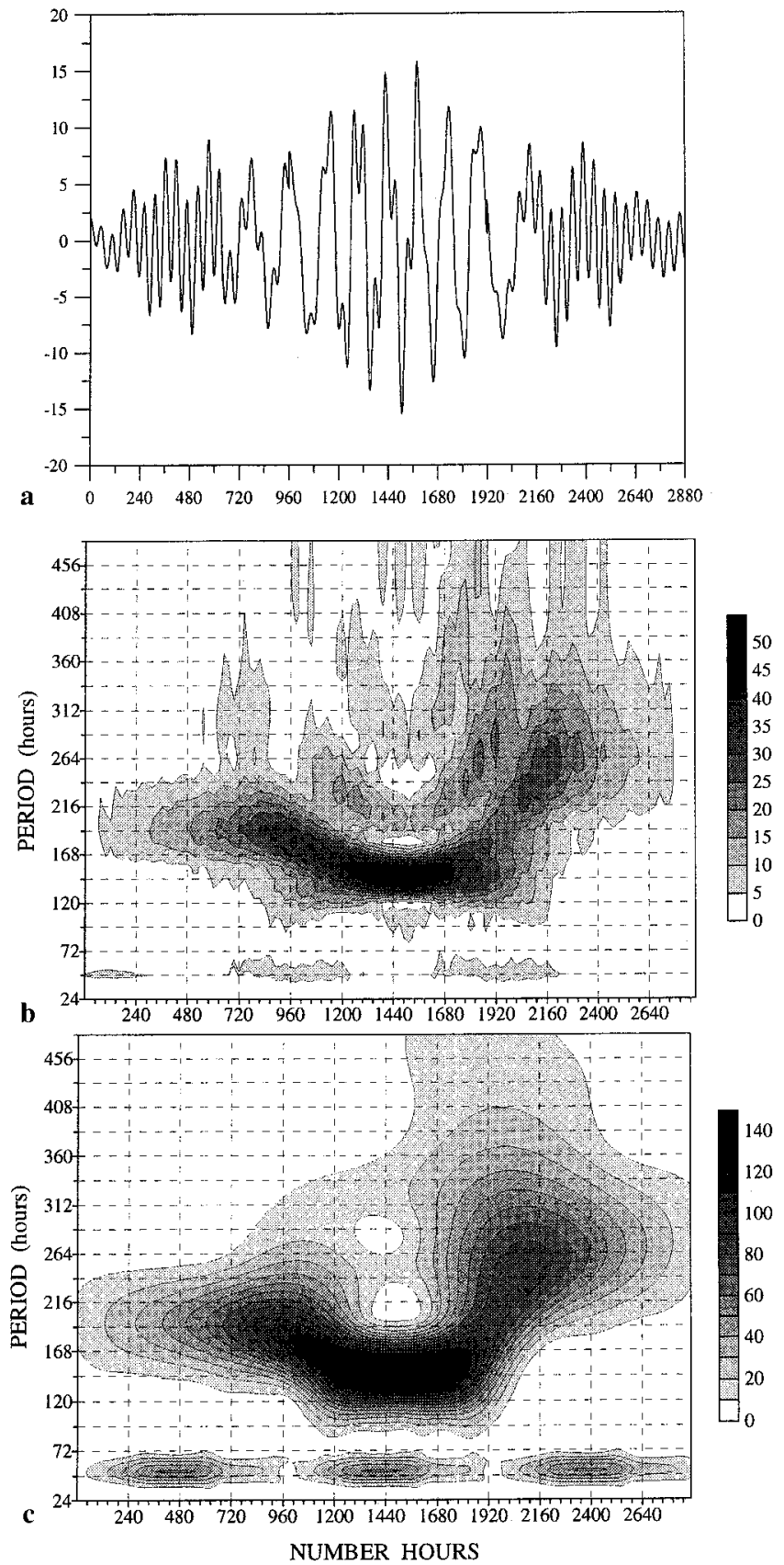

Fig. 6. a The simulated signal obtained as a sum of the defined by Eq. (8) wave packets. b The short-time Fourier transform performed on the simulated data, shown in a, with the length of the segment $720 \mathrm{~h}$ which is incremented in 12-h steps. c The Morlet wavelet transform of the simulated signal, presented in a 
$f(t)=\sum_{i=1}^{3} g_{i}(t)+\sum_{i=1}^{9} h_{i}(t)$

where:

$$
\begin{aligned}
g_{1}(t) & =\exp (0.002 t) \cos \left[\frac{2 \pi}{T_{1}}(t-3)\right] \quad t \in[0,1152] \\
g_{2}(t) & =10 \cos \left(\frac{2 \pi}{T_{2}} t\right) \quad t \in[1152,1872] \\
g_{3}(t) & =10 \exp (-0.0025 t) \cos \left[\frac{2 \pi}{T_{3}}(t+5)\right] \\
t & \in[1872,2880]
\end{aligned}
$$

as $T_{1}=192, T_{2}=144$ and $T_{3}=264 \mathrm{~h}$, and

$$
\begin{aligned}
& h_{1,4,7}(t)=\exp (0.0055 t) \cos \left(\frac{2 \pi}{52} t\right) \\
& t \in[0,320] ; \quad[960,1280] ; \quad[1920,2240] \\
& h_{2,5,8}(t)=5.8 \cos \left(\frac{2 \pi}{52} t\right) \\
& \quad t \in[320,640] ; \quad[1280,1600] ; \quad[2240,2560] \\
& h_{3,6,9}(t)=5.8 \exp (-0.001 t) \cos \left(\frac{2 \pi}{52} t\right) \\
& t \in[640,960] ; \quad[1600,1920] ; \quad[2560-2880] .
\end{aligned}
$$

The short-time Fourier transform is performed with the length of the segment $720 \mathrm{~h}$ and it is incremented in 12-h steps. The results from the short-time Fourier transform and Morlet wavelet transform are shown respectively on Fig. $6 \mathrm{~b}$ and c. The Morlet wavelet represents almost exactly the given frequency components and the times of their maxima. The short-time Fourier transform represents not bad the longer period waves (except some additional peak at about $216 \mathrm{~h}$ and secondary peak at $264 \mathrm{~h}$, which appears earlier than the given wave), but the short-time wave packets are completely dislocated. The short-time waves are centered at: $t=480,1440$ and 2400; this is clearly visible on Fig. 6c, but they totally absent at these times from the short-time Fourier transform (Fig. 6b). Consequently, the short-time Fourier transform can not represent adequately the signal with different periods, which occur at different time intervals.

The second example represents a signal containing three wave packets with two different central frequencies:

$$
\begin{aligned}
& f(t)=f_{1}(t)+f_{2}(t)+f_{3}(t) \\
& f_{1}(t)=\cos \left(\omega_{1} t+0.005 t^{2}\right) \quad t \in[0,240] \\
& f_{2}(t)=\cos \left(\omega_{2} t+0.0002 t^{2}\right) \quad t \in[240,480] \\
& f_{3}(t)=\cos \left[\omega_{1} t+\sin \left(\frac{2 \pi}{120} t\right)\right] \quad t \in[480,720]
\end{aligned}
$$

where $\omega_{1}=2 \pi / 24$ and $\omega_{2}=2 \pi / 16$. The result from the short-time Fourier transform performed by using a segment of length 96 which is incremented in steps of 6 , is shown on Fig. 7a. In general, the main behavior of the signal is shown, however the linear frequency modula-
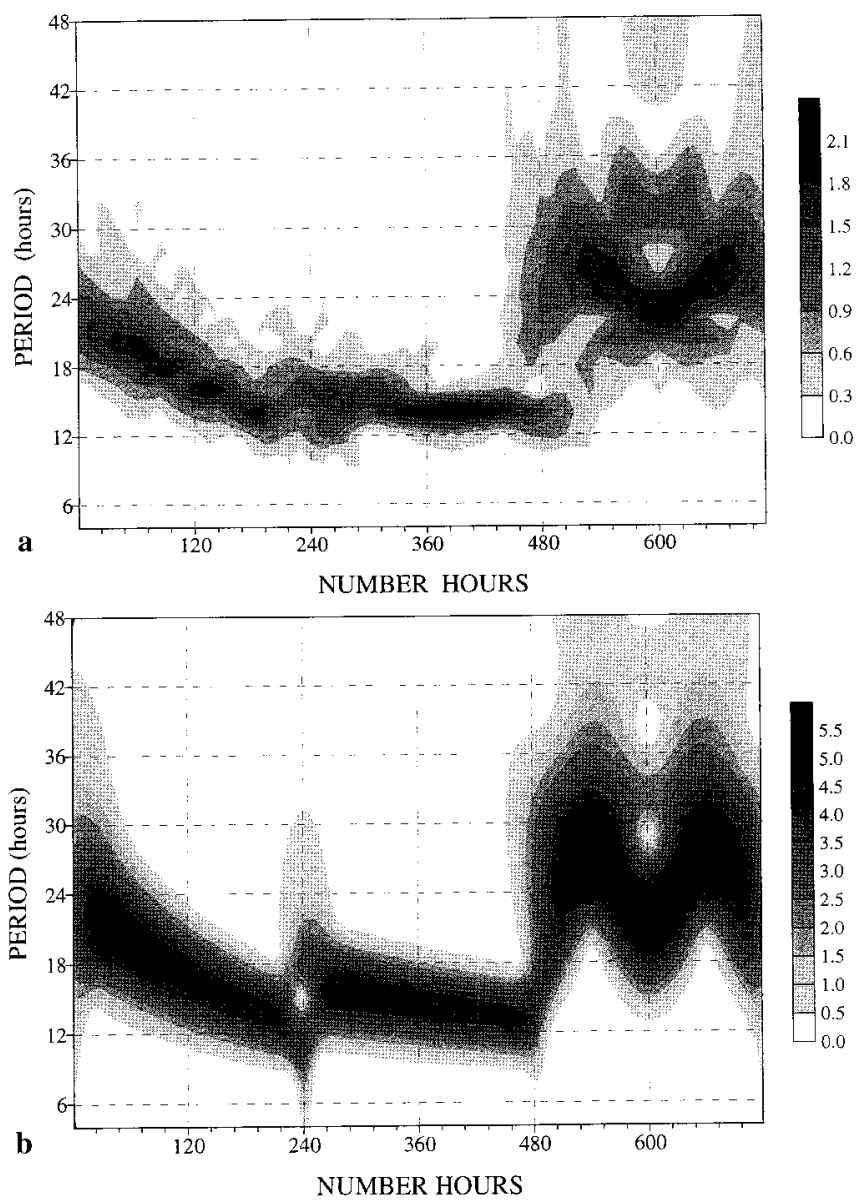

Fig. 7. a The short-time Fourier transform with the length of the segment 96, which is incremented in steps of 6, applied to the simulated signal, described by Eq. (9). b The Morlet wavelet transform of the same simulated signal

tion within the first two wave packets is represented by discrete consecutive spectral components with decreasing periods. When the linear trend is small (the second wave packet) two spectral components are evident with periods of 16 and 14 respectively. The last part of the signal, connected with a periodic frequency modulation of the basic wave with period 24 modulated by a wave with period equal to 120 , is represented by three maxima, corresponding to components with periods 29 and 20. The changes of the period from 29 to 20, and conversely, however is visible. Figure $7 \mathrm{~b}$ shows the wavelet transform of the signal, which represents exactly the given signal.

The last example is connected with the study of some irregular, wave-like event observed in the neutral wind of MLT region above Bulgaria. It is probably connected with the early final warming that started in the middle of March and intensified at the end of the month (Naujokat et al., 1991). Figure 8a shows such an anomaly, composed of longer and shorter oscillations in the zonal wind measured by meteor radar, which occurred in the second half of March 1991. The average duration of the longer oscillation is about 9-10 days, while the shorter 4-5 days. Figure $8 \mathrm{~b}$ presents the result from wavelet 
transform. Clearly outlined anomalies with mean periods of $\sim 5$ and 9 days are visible on the figure, and the maximum values of wavelet coefficients coincide with the strongest anomaly in the zonal wind, around 23-25 of March. Figure 8c shows the result obtained by shorttime Fourier transform using 60 days as the length of the
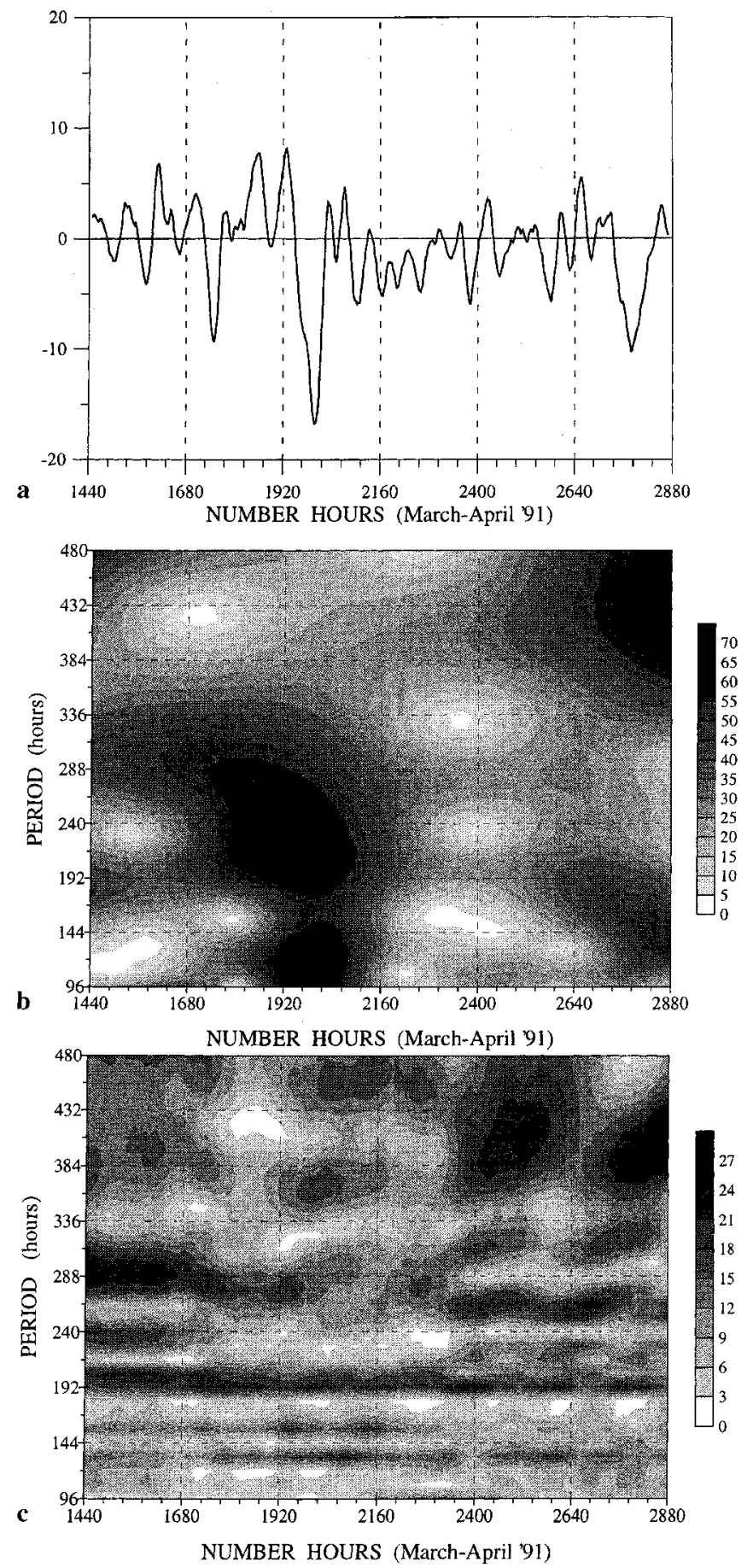

Fig. 8. a The hourly values of zonal neutral wind measured by meteor radar in the MLT region above Bulgaria in March-April, 1991. b The Morlet wavelet transform of the zonal neutral wind, shown in a. $\mathbf{c}$ The short-time Fourier transform with the length of the segment 60 days which is incremented in steps of $1 \mathrm{~h}$, applied to the same zonal neutral wind segment and a $1 \mathrm{~h}$ sliding interval (the wavelet transform, as well as the short-time Fourier transform of the zonal wind are initially performed for the interval 01 January-23 October 1991, and the results only for March-April, 1991 are displayed on Fig. 8b, c, so the edge-effect errors are excluded). There are no indications of fluctuations with periods 4-5 and 9-10 days in the second half of March. The reason is that the Fourier transform does not lose the information, but instead "spreads" the information about the localized wave event over all scales in the Fourier space.

The above results indicated that the Morlet wavelet transform is convenient for investigating signals with: (1) several different spectral components which may occur at different time intervals; (2) amplitude and frequency modulated spectral components; and (3) separating singular, wave-like events, observed in the neutral wind of MLT region, related usually to large scale disturbances forcing from below. Consequently, this is the tool needed to study the transient behavior of the tidal variability observed by meteor radar in the MLT region above Bulgaria.

\section{Wavelet analysis of tidal variability}

The semidiurnal and diurnal tides are the basic tidal components in the dynamics of the MLT region above Bulgaria. So, to study the transient behavior of the tidal variability the wavelet transform is applied only to the time series of these mentioned tidal amplitudes. Figure $9 \mathrm{a}$ shows the wavelet transform of the semidiurnal tidal amplitudes in the zonal neutral wind. The oscillations with the following periods are outlined: $2-3,4-5$, 8-10 and 16-17 days. In this work, like Jacobi et al. (1998), we refer to the quasi 16-day wave as oscillation in the period range between about 12-13 and 20 days, and therefore keep the term "16-day wave" even if the measured period differs. The temporal variation of the periods of some waves is clearly followed by the wavelet analysis. For example, the 16-day wave has a prevailing period of 16 days in January and February 1991, but at the end of March and April it becomes 12-13 days and later, in May and June it again approaches 16 days. In autumn the period of the quasi-16-day wave increases to or above 20 days, but in January and February 1992, it is about 15 days. In June 1992 there is an indication that the summer 16-day wave may appear, but unfortunately the measurements finished on 16 June. The period of $\sim 8$-day oscillation also changes during the time; in summer it is 8 days, but in autumn and winter it splits into two oscillations with main periods $7-8$ and $10-11$ days. Figure 9b shows the analogous result to Fig. 9a, but for the amplitudes of the diurnal tide in the zonal neutral wind. The quasi-16-day wave observed in the amplitudes of the diurnal tide and the semidiurnal tide are both stronger in the first subinterval. Its prevailing period is about 17-18 days in March-April 1991, then decreases to $\sim 15$ days in May and at the end of summer and autumn (similarly to the analogous oscillation in the semidiurnal tide), the period increases to or above 20 

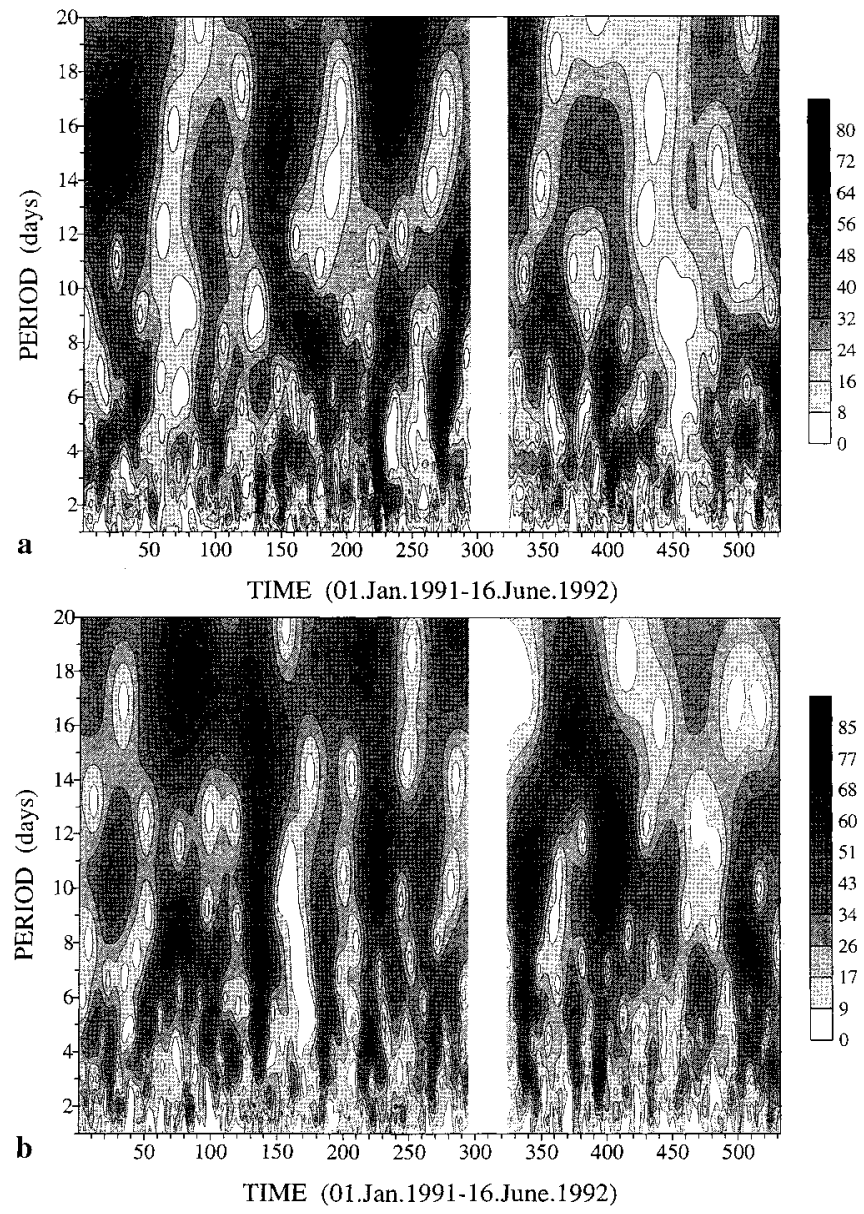

Fig. 9. a The Morlet wavelet transform of the amplitudes of semidiurnal zonal tide for the interval January 1991-June 1992. b The same as Fig. 4a, but for the amplitudes of diurnal zonal tide

days. In January-February 1992, the prevailing period is 16 days. A strong 10-day wave is observed in the amplitude of the diurnal zonal tide. The prevailing period is almost stable, excluding March-May 1991, when it decreases to about 8 days. The well-developed 4-5-day oscillations are also visible during the whole interval under consideration.

Figure 10a represents the development of the planetary waves in the amplitudes of the semidiurnal meridional tide. The strongest and most regular wave observed in the whole time series is again the quasi-16day wave. In January 1991, its prevailing period is 14 days, but from the end of February to the end of April it increases to 16 days. At the end of the summer and the beginning of autumn, the period again increases to 18 days, but in December 1991, it decreases to about 17 days. From January 1992, to the end of the measurements, this oscillation is strongly amplified, with a prevailing period of $\sim 14$ days. The periodic frequency modulation, similar to that expressed in the second synthetic time series (the third wave packet of Fig. 7b) can be seen. The modulation period is about 60 days. There is also a well-expressed 8-day wave, observed in March-April 1991 and February-March 1992, when the period is almost stable, whilst in summer, June-August
1991 it changes from 8 to 10 days. Again the 4-5-day wave can be seen, as well as the quasi-2-day wave, which is known to be a regular summer event for the neutral wind. In the amplitudes of the semidiurnal meridional tide there is a quasi-2-day event in winter in addition to the usual summer event (later we will see the same in the amplitudes of the diurnal meridional tide). Pancheva and Mukhtarov (1994) indicated that the largest amplitudes of the quasi-2-day wave in the neutral wind of the MLT region above Bulgaria are observed during midsummer (July-August) and a smaller maximum during winter. Figure $10 \mathrm{~b}$ shows the planetary waves in the amplitudes of diurnal meridional tide. The planetary wave activity for $\sim 10$ - and quasi-16-day waves are definitely stronger in the second subinterval. The quasi-2 and 3-4-day waves are seen in both subintervals. There are two pulses of the quasi-16-day wave in spring and summer of 1991 with a mean period of 14 days. In December 1991-January 1992 its prevailing period is 16 days, but in February and March when this wave amplifies, the period decreases to 14 days. In June, evidence for a new 12-13-day wave can be distinguished. A 7-9-day wave can be outlined also.
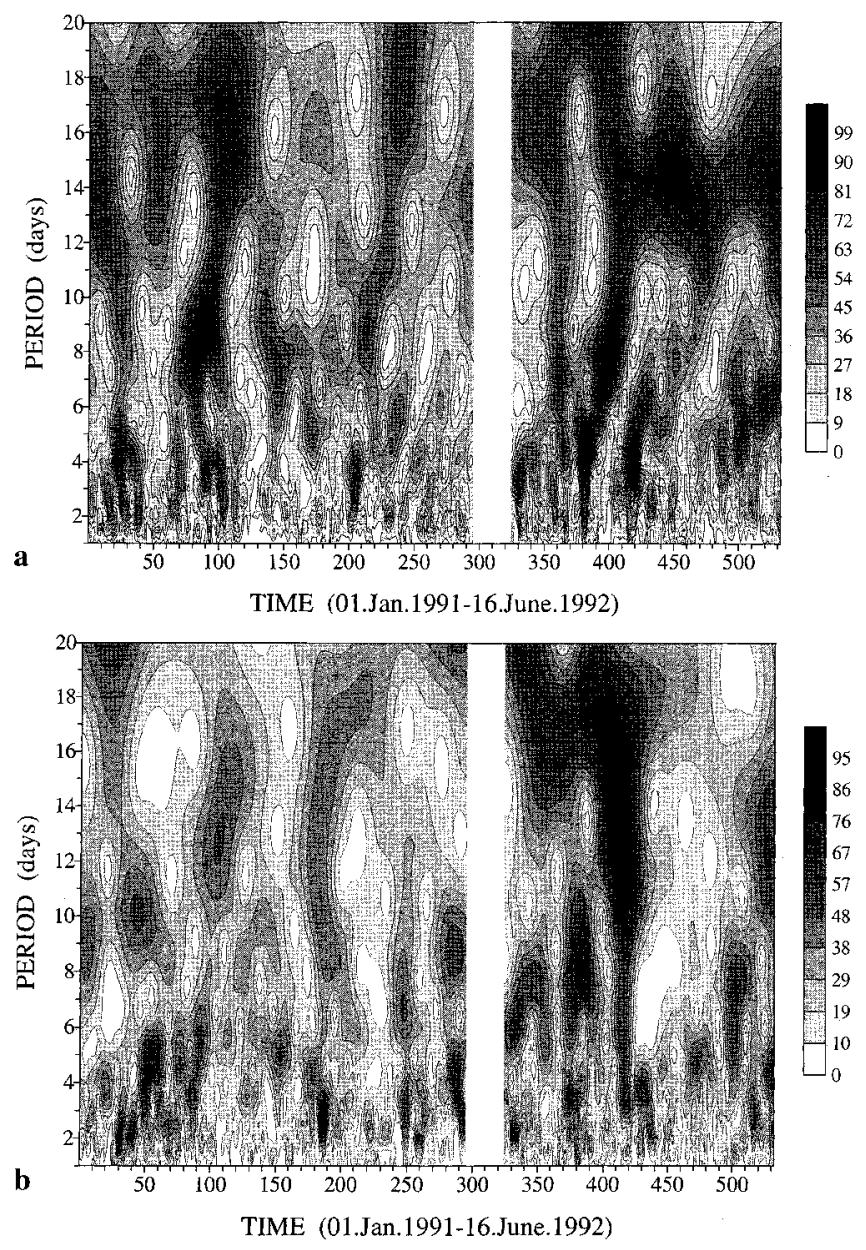

Fig. 10. a The Morlet wavelet transform of the amplitudes of semidiurnal meridional tide for the interval January 1991-June 1992. b The same as a, but for the amplitudes of diurnal meridional tide 

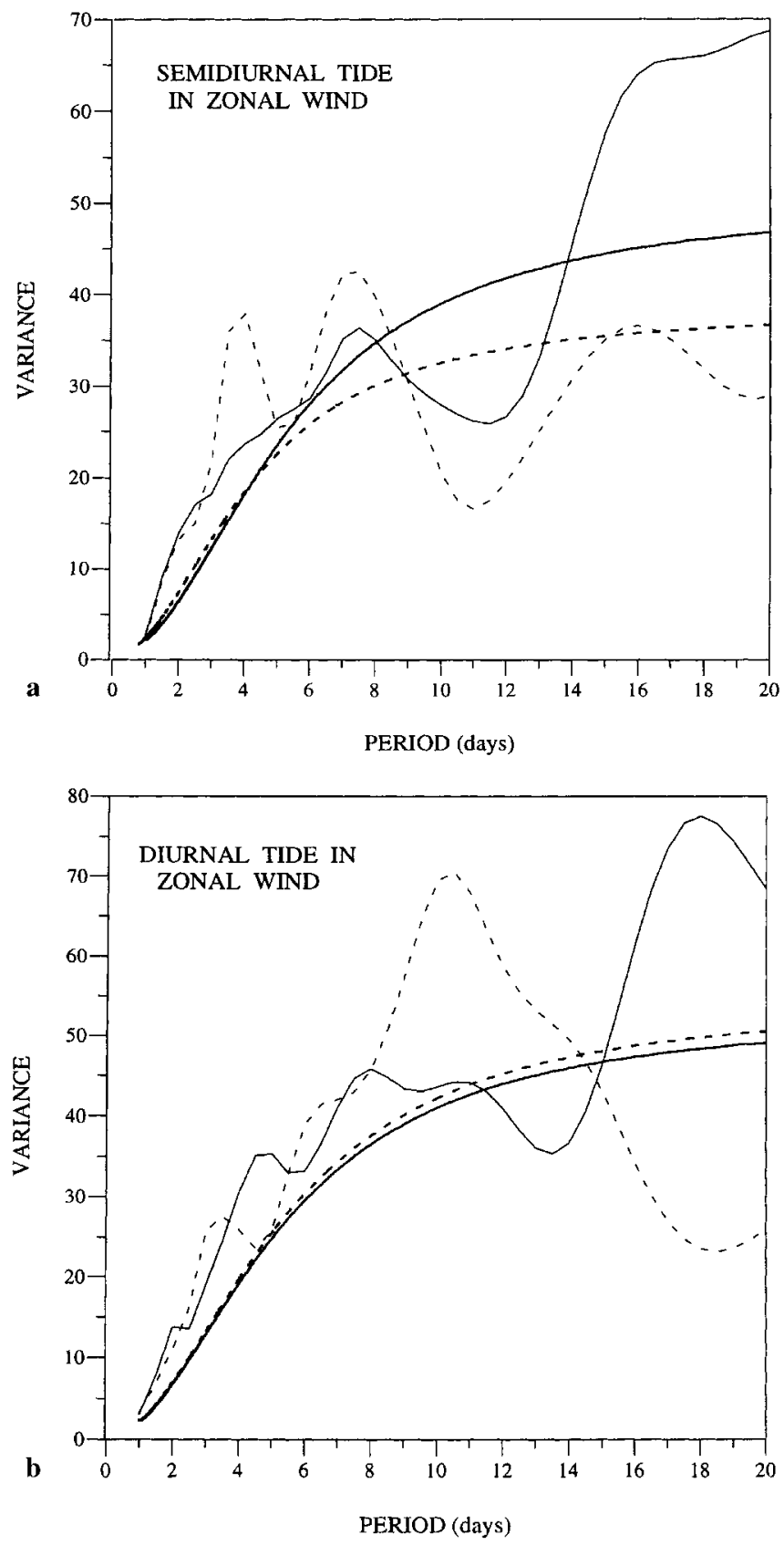

Fig. 11. a The wavelet power spectra, normalized by the variance of the time series, for the amplitudes of the semidiurnal zonal tide (solid line, for the first subinterval, dashed line, for the second subinterval). The thick solid (dashed) line represents the 95\% confidence level for the first (second) subinterval of the measurements. b The same as a, but for the amplitudes of diurnal zonal tide

One of the most widely used tools of Fourier analysis is the Parseval theorem which indicates how the energy in the signal is distributed among frequencies. In Sect. 3.1 it was shown that a similar conservation of energy theorem is valid for the wavelet transform and the wavelet energy spectrum can be obtained by this theorem (see Eqs. 4 and 5). To make it easier to compare different wavelet power spectra, it is desirable to find a common normalization for the wavelet spectrum. Torrence and Compo (1998) indicated that for a white-noise process the wavelet power spectrum is $|W(a, b)|^{2}=\sigma^{2}$ at all $a$ and $b$, where $\sigma^{2}$ is the variance of the time series. In this way, the normalization by $1 / \sigma^{2}$ gives a measure of the power relative to white noise. Figure 11a shows the wavelet power spectrum, normalized by the variance of the time series, for the amplitudes of the semidiurnal zonal tide. The two subintervals are represented by solid and dashed lines respectively. The thick solid (dashed) line represents the 95\% confidence level for the first (second) subinterval wavelet power spectrum. To determine significance levels, first we need to choose an appropriate background spectrum. In the present work a red-noise spectrum is used to establish a null hypothesis for the significance of a peak in the wavelet power spectrum. A theoretical formula, described in Gilman et al. (1963) calculate it. Torrence and Compo (1998) pointed out that the distribution of the wavelet power spectrum is: $\left(|W(a, b)|^{2} / \sigma^{2}\right) \Rightarrow \frac{1}{2} P_{k} \chi_{2}^{2}$ at each time $b$ and scale $a$ (this is true for the continuous wavelet transform, but in the case of the discrete wavelet transform there must be some minimum distance between each $a$ and $b$ to ensure independence, otherwise the number of degrees of freedom for the chi-squared distribution will not be 2). The value of $P_{k}$ is the accepted background spectrum at the Fourier frequency $k$ that corresponds to the wavelet scale $a$ and $\chi_{2}^{2}$ is the chi-square distribution with two degrees of freedom. The red-noise background spectra, used for assessment of the $95 \%$ confidence level in Fig. 11a, are calculated for $\alpha=0.875$ (first subinterval) and $\alpha=0.84$ (second subinterval), as $\alpha=\left(\alpha_{1}+\sqrt{\alpha}_{2}\right) / 2$ where $\alpha_{1}$ and $\alpha_{2}$ are lag-1 and lag-2 autocorrelation coefficients of the respective time series. Similar peaks higher than 95\% confidence level can be seen in the wavelet power spectra from Fig. 11a corresponding to 4-5, 8 and 16-17 days. Figure $11 \mathrm{~b}$ shows the power spectra for the amplitudes of the diurnal zonal tide. The red-noise background spectra for the first and second subintervals are calculated for $\alpha=0.885$ and $\alpha=0.888$ respectively. The strongest peaks in the first and second subintervals are respectively $17-18$ days and $\sim 10$ days. Some similarity can be seen between the power spectra of diurnal and semidiurnal zonal tides in the first subinterval.

Figure 12a shows the wavelet power spectra for the amplitudes of the semidiurnal meridional tide. The rednoise background spectra for the first and second subintervals are calculated for $\alpha=0.855$ and $\alpha=0.901$ respectively. Peaks, higher than $95 \%$ confidence level, are situated at 2, 4-5, 8 and 17 days for the first subinterval and 14 days for the second subinterval. Figure $12 \mathrm{~b}$ represents the wavelet power spectra for the amplitudes of the diurnal meridional tide. The red-noise background spectra are calculated for $\alpha=0.776$ and $\alpha=0.88$. The main power in the first subinterval is distributed in the short-period waves (2-6 days), while in the second subinterval the power is distributed in longerperiod waves (16-17 days). Figure 12 also shows that the variability in the amplitudes of the diurnal and semidiurnal meridional tides is stronger in the second subinterval and that this is related to the amplified long-period waves with periods of 16-17 and 14 days respectively. 

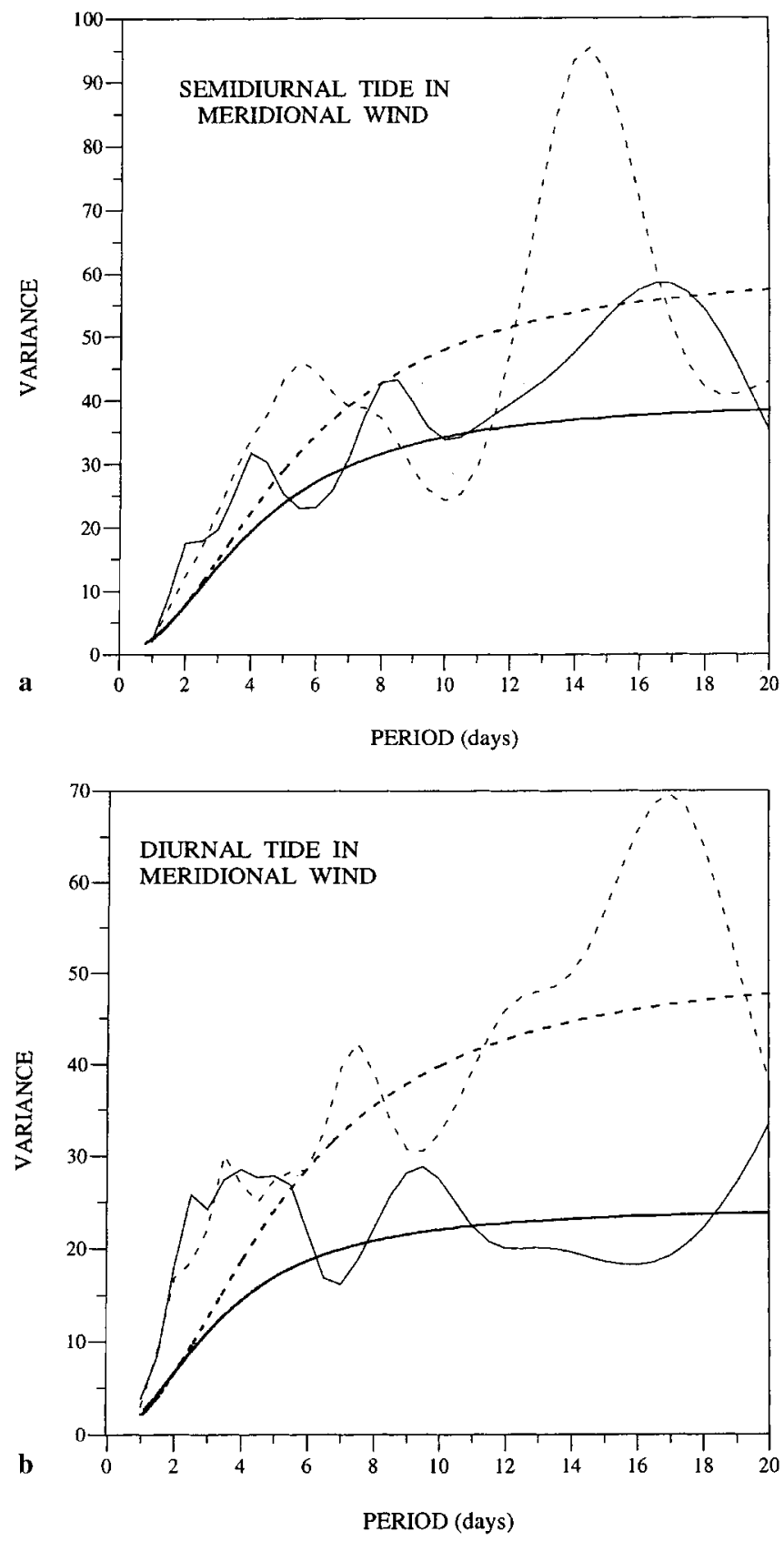

Fig. 12. a The same as Fig. 11a, but for the amplitudes of the semidiurnal meridional tide. b The same as a, but for the amplitudes of the diurnal meridional tide

These results obtained can be summarized as follows: (1) the tidal variability with periods of planetary waves reveals similar periodicities: 2, 4-5, 8-10 and 14-18 days; and (2) the time development, however, of these planetary waves observed in the amplitudes of the diurnal and semidiurnal tides demonstrate some differences.

\section{Discussion and conclusions}

On the basis of hourly data set of neutral wind measured by meteor radar in the MLT region above Bulgaria and application of bispectral analysis it was demonstrated that nonlinear processes are frequently and regularly acting in the mesopause region. They contribute significantly to the short-term tidal variability and are apparently responsible for its complicated behavior. A Morlet wavelet transform is proposed as a technique for studying nonstationary signals. By simulated data it was demonstrated that the wavelet transform could represent adequately the given signal and is definitely better technique than short-time Fourier transform, widely used in the atmospheric studies. The Morlet wavelet transform is especially convenient for investigating signals with: (1) several different spectral components with complicated temporal behavior; (2) amplitude and frequency modulated spectral components; and (3) singular, wave-like events, observed in the neutral wind of the MLT region, related usually to large-scale disturbances forcing from below. In the time series of neutral mesospheric wind or the amplitudes and phases of the atmospheric tides all the peculiarities mentioned of the signals can be found. Usually the tidal amplitudes display a wide range of dominant frequencies, which are localized in different time intervals. The main advantage of using a wavelet transform is that it preserves the information about the local features of the signal, while a Fourier transform spreads information about the localized features over all scales in the Fourier space.

On the basis of Morlet wavelet transform applied to the hourly values of the amplitudes of diurnal and semidiurnal tides the tidal variability with periods of planetary waves (1.5-20 days) observed in the MLT region above Bulgaria during the interval January 1991June 1992, were studied. It was found that all time series under consideration reveal the similar periodicities: $\sim 2$, 4-5, 8-10 and 14-18 days. Their temporal development, however, demonstrated some differences. In general, the similarity between the variability of the diurnal and semidiurnal tidal amplitudes is closer in the zonal component than in the meridional one. The zonal wind measured by meteor radar above Bulgaria is definitely stronger that the meridional wind. This could be a reason for stronger interaction between the mean zonal wind and the tides, as well as between the planetary waves present in the neutral wind and atmospheric tides.

To study the dominant periodicities simultaneously observed in the neutral wind and tidal amplitudes we use cross-wavelet spectrum. If two time series $X$ and $Y$ are given, with wavelet transforms $W^{X}(a, b)$ and $W^{Y}(a, b)$, one can define the cross-wavelet spectrum as: $W^{X Y}(a, b)=W^{X}(a, b) W^{Y^{*}}(a, b)$, where $(*)$ again means the complex conjugate. The cross-wavelet spectrum is complex number and the cross-wavelet power can be defined as: $\left|W^{X Y}(a, b)\right|$.

Figure 13a shows the cross-wavelet spectrum between the mean zonal wind and the amplitudes of the semidiurnal zonal tide, while Fig. $13 \mathrm{~b}$ represents the same, but for the amplitudes of the diurnal zonal tide. Both figures clearly outline the similar time intervals of increased planetary wave activity simultaneously in the zonal wind and the amplitudes of diurnal and semidiurnal zonal tides. The figures show the main oscillations with periods of planetary waves (1.5-20 days) existing 

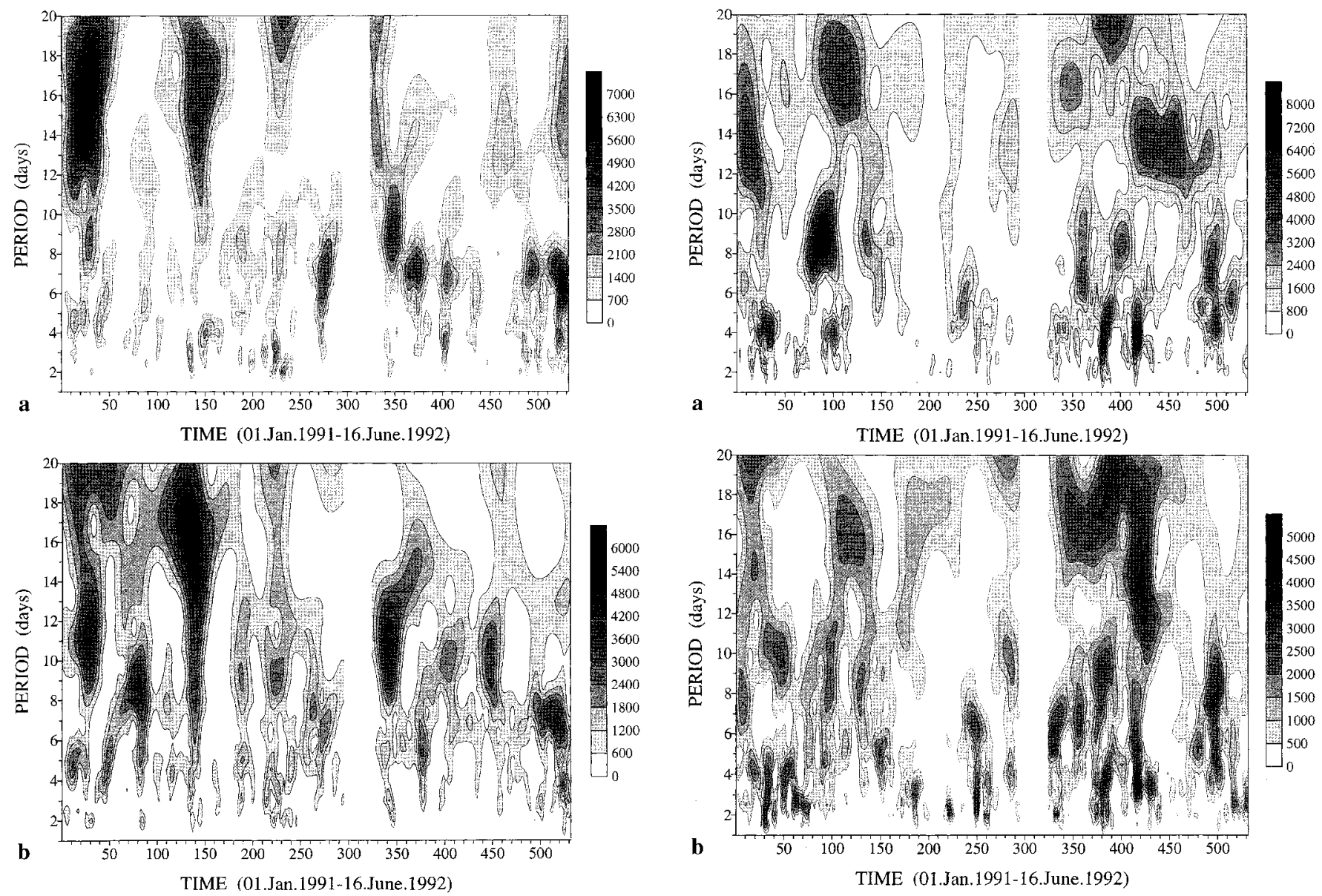

Fig. 13a, b. The cross-wavelet spectra obtained between: a the mean zonal wind and the amplitudes of the semidiurnal zonal tide, and $\mathbf{b}$ the mean zonal wind and the amplitudes of the diurnal zonal wind

simultaneously in the prevailing zonal wind and the amplitudes of semidiurnal and diurnal tidal velocities, as well as the time intervals of their localizing. In the first subinterval the well-expressed oscillations observed simultaneously in the zonal wind and in the amplitudes of semidiurnal tides are (Fig. 13a): $\sim 2, \sim 5,8-10$ and quasi-16 days. The bispectral result (not shown here) indicates a quadratic nonlinear interaction between the semidiurnal tide and the above mentioned planetary waves. The bispectral estimate shown on Fig. 5a describes the nonlinear interaction between tides and planetary waves during the second subinterval. The semidiurnal tide in the zonal wind interacts mainly with 7 and 10-12-day planetary waves. The oscillations with these periods can be clearly outlined in Fig. 13a during the second subinterval. Therefore, if we assume that the non-linear coupling is a local process, then Fig. 13a clarifies the time intervals when the nonlinear interactions between the semidiurnal tide and the mentioned planetary waves take place. Figure $13 \mathrm{~b}$ shows the oscillations with periods: $\sim 2,4-6,8-10$ and 16 days, observed simultaneously in the amplitudes of the diurnal tide and the prevailing zonal wind during the first subinterval. The bispectral result (not shown here) indicates weak nonlinear interactions of the diurnal

Fig. 14a, b. The cross-wavelet spectra obtained between: a the mean meridional wind and the amplitudes of the semidiurnal meridional tide, and $\mathbf{b}$ the mean meridional wind and the amplitudes of the diurnal meridional tide

tides only with the 5- and 16-day planetary waves. The origin of the strong 8-10-day oscillation in the amplitudes of the diurnal zonal tide (see Fig. 9b also) during the first subinterval remains unclear now. The welloutlined oscillations observed simultaneously in the amplitudes of diurnal zonal tide and the neutral zonal wind during the second subinterval are: $\sim 2,7-8$ and 10 12-day ones. The bispectral result, shown on Fig. 5a proves that these oscillations observed in the amplitudes of the diurnal zonal tide are a result from the nonlinear interaction with the respective planetary waves and Fig. 13b outlined their time intervals if these nonlinear processes are local.

Figure 14a represents the cross-wavelet spectrum between the prevailing meridional wind and the amplitudes of semidiurnal meridional tide, while Fig. 14b shows the same, but for the amplitudes of the diurnal meridional tide. In the first subinterval the oscillations with periods: $\sim 2,4,8-10,13-14$ and 16-18 days are observed simultaneously in the neutral meridional wind and the semidiurnal tide (Fig. 14a). The bispectral estimate, shown on Fig. $4 \mathrm{a}$, reveals strong nonlinear interactions between the semidiurnal meridional tide and all the mentioned planetary waves, with an exception related to the 13-14-day planetary wave. In the second 
subinterval the oscillations with periods: $\sim 2,3-5,7-8$ and 14-16 are well outlined. The bispectral estimate, shown on Fig. 5b, indicates nonlinear interactions between the semidiurnal meridional tide and the above mentioned planetary waves, except 7-8-day wave. The oscillations with periods: $\sim 2,3-5,7-8,10-12$ and $\sim 16$ days are observed simultaneously in the meridional wind and the amplitudes of the diurnal meridional tide. The bispectrum estimate, shown on Fig. 4a, reveals that only the 16-day wave does not participate in the nonlinear coupling between the diurnal tide and the planetary waves. In the second subinterval only the $\sim 2$ and 4-day oscillations observed in the amplitudes of diurnal tide (and simultaneously in the meridional wind also) could be a result from the nonlinear interaction processes (Fig. 5a). The origins of all the rest clearly visible oscillations: 6-7, 9-10, 14 and 18-19 days, are not clear.

Using a cross-wavelet analysis an attempt was made to clarify the origin of the observed tidal variations with periods of planetary waves (1.5-20 days). The results obtained give an additional support to the idea that the processes of nonlinear interactions between different waves in the MLT region are a significant source of the tidal variability. The results of bispectral analysis indicate which planetary waves participated in the process of nonlinear coupling with the atmospheric tides, while the results of crosswavelet analysis outline their time intervals if these interactions are local. Some of the oscillations observed mainly in the amplitudes of the diurnal tide probably are not a result of local interaction and their origin has to be cleared up.

Acknowledgements. Topical Editor F. Vial thanks S. Palo and F. Kamalabadi for their help in evaluating this paper.

\section{References}

Apostolov, E. M., D. Altadill, and L. Alberca, Characteristics of quasi-2-day oscillations in the foF2 at northern middle latitudes, J. Geophys. Res., 100, 12163-12171, 1995.

Avery, S., R. Vincent, A. Phillips, A. Manson, and G. Fraser, High latitude tidal behavior in the mesosphere and lower thermosphere, J. Atmos. Terr. Phys., 51, 595-608, 1989.

Beard, A. G., N. Mitchell, P. J. S. Williams, W. Jones, and H. Muller, Mesopause-region tidal variability observed by meteor radar, Adv, Space Res., 6, 1237-1240, 1997.

Beard, A. G., N. Mitchell, P. J. S. Williams, and M. Kunitake, Nonlinear interactions between tides and planetary waves resulting in periodic tidal variability, J. Atmos. Sol.-Terr. Phys., 61, 363-376, 1999.

Bremer, J., R. Schminder, K. Greisiger, P. Hoffmann, D. Kürschner, and $\mathbf{W}$. Singer, Solar cycle dependence and long-term trends in the wind field of the mesosphere/lower thermosphere, J. Atmos. Sol.-Terr. Phys., 59, 497-509, 1997.

Brillinger, D., and M. Rosenblatt, Asymptotic theory of kth order spectra in Spectral analysis of time series, Ed. B. Haris, Wiley, New York, 1967, pp 153-188.

Carter, D., and B. Balsley, The summer wind field between 80 $93 \mathrm{~km}$ observed by the MST radar at Poker Flat, Alaska $\left(65^{\circ} \mathrm{N}\right)$, J. Atmos. Sci., 39, 2905-2915, 1982.

Cevolani, G., and S. Kingsley, Non-linear effects on tidal and planetary waves in the lower thermosphere: preliminary results, Adv. Space Res., 12(10), 77-83, 1992.
Clark, R., and J. Bergin, Bispectral analysis of mesosphere wind, J. Atmos. Sol.-Terr. Phys., 59, 629-639, 1997.

Darrt, D., G. Nastrom, and A. Belmont, Seasonal and solar cycle wind variations, 80-100 km, J. Atmos. Terr. Phys., 45, 707-718, 1983.

Daubechies, I., The wavelet transform, time-frequency localization and signal analysis, IEEE Trans. Inf. Theory, 36(5), 961-1005, 1990.

Elgar, S., and R. T. Guza, Statistics of bicoherence, IEEE Trans. on Acoustics, Speech. and Signal Processing, 36, 1667-1668, 1988.

Espy, P., J. Stegman, and G. Witt, Interannual variations of the quasi 16-day oscillation in the polar summer mesospheric temperature, J. Geophys. Res., 102, 1983-1990, 1997.

Farge, M., Wavelet transforms and their applications to turbulence, Ann. Rev. Fluid. Mech., 24, 395-457, 1992.

Forbes, J., and M. Hagan, Diurnal propagating tide in the presence of mean winds and dissipation: a numerical investigation, Planet. Space Sci., 36, 579-590, 1988.

Forbes, J., and F. Vial, Monthly simulation of the solar semidiurnal tide in the mesosphere and lower thermosphere, J. Atmos. Terr. Phys., 51, 649-662, 1989.

Fritts, D. C., A review of gravity saturation processes, effects and variability in the middle atmosphere, Pure Appl. Geophys., 130, 343-371, 1989.

Fritts, D., and R. Vincent, Mesospheric momentum flux studies at Adelaide, Australia: observations and a gravity wave-tidal interaction model, J. Atmos. Sci., 44, 605-619, 1987.

Gabor, D., Theory of communications, J. IEE, 93(3), 429-457, 1946.

Gilman, D., F. Fuglister, and J. Mitchell, On the power spectrum of "red noise", J. Atmos. Sci., 20, 182-184, 1963.

Greisiger, K., R. Schminder, and D. Kürschner, Long-period variations of wind parameters in the mesopause region and the solar cycle dependence, J. Atmos. Terr. Phys., 49, 281-285, 1987.

Hagan, M., F. Vial, and J. Forbes, Variability in the upward propagating semidiurnal tide due to effect of QBO in the lower atmosphere, J. Atmos. Terr. Phys., 54, 1465-1474, 1992.

Hagan, M., J. Forbes, and F. Vial, On modelling migrating solar tides, Geophys. Res. Lett., 22, 893-896, 1995.

Haubrich, R. A., Earth noise, 5 to 500 millicycles per second: 1. Spectral stationarity, normality, and nonlinearity, J. Geophys. Res., 70, 1415-1427, 1965.

Huuskonen, A., T. Virdi, G. Jones, and P. J. S. Williams, Observations of day-to-day variability in the meridional semidiurnal tide at $70^{\circ} \mathrm{N}$, Ann. Geophysicae, 9, 407-415, 1991.

Jacobi, C., The quasi 16-day wave in the summer midlatitude mesopause region and its - dependence on the equatorial quasibiennial oscillation, Report of the LIM and the IFT9, Leipzig, 117-129, 1998.

Jacobi, C., R. Schminder, D. Kürschner, J. Bremer, K. Greisiger, P. Hoffmann, and W. Singer, Long-term trends in the mesosphere wind field obtained from D1 LF wind measurements at Collm, Germany, Adv. Space Res., 20, 2085-2088, 1997.

Jacobi, C., R. Schminder, and D. Kürschner, Non-linear interaction of the quasi-2-day wave and long-term oscillations in the summer midlatitude mesopause region as seen from LF D1 wind measurements over Central Europe $\left(\right.$ Collm, $\left.52^{\circ} \mathrm{N}, 15^{\circ} \mathrm{E}\right)$, J. Atmos. Sol.-Terr. Phys., 60, 1175-1191, 1998.

Kamalabadi, F., J. Forbes, N. Makarov, and Y. Portnyagin, Evidence for non-linear coupling of planetary waves and tides in the Antarctic mesopause, J. Geophys. Res., 102, D4, 44374446, 1997.

Kim, Y. C., and E. J. Powers, Digital bispectral analysis and its application to nonlinear wave interactions, IEEE Trans. Plasma Science, PS-7(2), 120-131, 1979.

Kopecky, M., and G. Kuklin, About 11-year variation of the mean life duration of a group solar spots, Issled. Geomagn. Aeronom. Fiz. Solntsa, 2, 167, 1971 (in Russian).

Lii, K. S., and K. N. Helland, Cross-bispectrum computation and variance estimation, ACM Trans. Math. Software, 7, 284-294, 1981. 
Lii, K. S., M. Rosenblatt, and C. Van Atta, Bispectral measurements in turbulence, J. Fluid Mech., 77, 45-62, 1976.

Luzov, A., G. Kuklin, and A. Petemsky, Investigation of the periodic variations of the cosmic ray intensity with the help of selected frequency filteres, Geomag. Aeronom., 5, 392, 1965 (in Russian).

Lysenko, I. A., B. Petrov, H. V. Spassov, and P. Manev, Wind measurements by meteor radar method in BPK, Bulg. Geophys. $J .$, 14, 34-47, 1988 (in Russian).

Ma, S., and K. Schlegel, Non-linear wave-wave interactions related to gravity wave reflection in the auroral upper F-region observed with the EISCAT radar, J. Atmos. Terr. Phys., 55, 719-738, 1993.

Manson, A., and C. Meek, Long-period ( $\sim 8-10$ h) wind oscillations in the upper middle atmosphere at Saskatoon $\left(52^{\circ} \mathrm{N}\right)$ : evidence for nonlinear tidal effects, Planet. Space Sci., 38, 1431-1441, 1990.

Manson, A., C. Meek, J. Gregory, and D. Chakrabarty, Fluctuations in tidal (24-, 12-h) characteristics and oscillations (8-h, $5-\mathrm{d})$ in the mesosphere and lower thermosphere $(70-110 \mathrm{~km})$, Saskatoon 1979-1981, Planet. Space Sci., 30, 1283-1294, 1982.

Manson, A., C. Meek, H. Teitelbaum, F. Vial, R. Schminder, D. Kürschner, M. Smith, G. Fraser, and R. Clark, Climatologies of the semidiurnal and diurnal tides in the middle atmosphere $(70-100 \mathrm{~km})$ at middle latitudes $\left(45-55^{\circ}\right)$, J. Atmos. Terr. Phys., 51, 579-594, 1989.

Meyer, Y., Wavelets and applications, Springer, New York Berlin Heidelberg, 1992.

Mitchell, N., P. J. S. Williams, A. G. Beard, G. Buesnel, and H. Muller, Non-linear planetary/tidal wave interactions in the lower thermosphere observed by meteor radar, Ann. Geophysicae, 19, 364-366, 1996.

Mukhtarov, P., and D. Pancheva, A new approach in studying of the neutral wind tides, Comp. Rend. Bulg. Acad. Sci., 46, 59-62, 1993.

Namboothiri, S., A. Manson, and C. Meek, Variations of mean winds and tides in the upper middle atmosphere over a solar cycle, Saskatoon, Canada, $52^{\circ} \mathrm{N}, 107^{\circ} \mathrm{W}$, J. Atmos. Terr. Phys., 55, 1325-1334, 1993.

Naujokat, B., K. Labitzke, R. Lenschow, K. Petzoldt, B. Rajewski, and R.-C. Wohlfart, The strarospheric winter 1990/91: a major midwinter warming as expected, Beilage zur Berliner Wetterkarte, SO13/91, 1991.

Nikias, C., and M. Radhuveer, Bispectrum estimation: a digital signal processing framework, Proc. IEEE, 75, 869-891, 1987.

Palo, S. E., R. G. Roble, and M. E. Hagan, TIME-GCM results for the quasi-2-day wave, Geophys. Res. Lett., 25, 3783-3786, 1998.

Palo, S. E., R. G. Roble, and M. E. Hagan, Middle atmosphere effects of the quasi-two-day wave determined from a general circulation model, Earth Planet. Space, 51, 629-647, 1999.

Pancheva, D., Evidence for non-linear coupling of planetary waves and tides in the lower thermosphere over Bulgaria, J. Atmos. Sol.-Terr. Phys., 1999 (in press).
Pancheva, D., and P. Mukhtarov, Variability of the mesospheric dynamics observed in Yambol by meteor radar, J. Atmos. Terr. Phys., 56, 1271-1278, 1994.

Pancheva, D., and P. Mukhtarov, Seasonal model of dynamical regime in the upper mesosphere over Bulgaria, Bulg. Geophys. $J ., 22,31-41,1996$ (in Bulgarian).

Pancheva, D., and P. Mukhtarov, Observed response of the ionospheric F2-layer and lower thermosphere dynamics to geomagnetic storms during high solar activity, Stud. Geoph. Geodet., 42, 119-136, 1998.

Pancheva, D., L. F. Alberca, and B. A. de la Morena, Simultaneous observation of the quasi-two-day variations in the lower and upper ionosphere over Europe, J. Atmos. Terr. Phys., 56, 43-50, 1994.

Pancheva, D., A. G. Beard, N. J. Mitchell, and H. G. Muller, Nonlinear interactions between planetary waves in the mesosphere/lower-thermosphere region, J. Geophys. Res., 1999 (in press).

Rüster, R., VHF radar observations in the summer polar mesosphere indicating non-linear interaction, Adv. Space Res., 12(10), 85-88, 1992.

Rüster, R., VHF radar observations of nonlinear interactions in the summer polar mesosphere, J. Atmos. Terr. Phys., 56, 12891299, 1994.

Sprenger, K., and R. Schminder, Solar cycle dependence of winds in the lower ionosphere, J. Atmos. Terr. Phys., 31, 217-221, 1969.

Teitelbaum, H., and F. Vial, On tidal variability induced by nonlinear interaction with planetary waves, J. Geophys. Res., 96, A8, 14169-14178, 1991.

Teitelbaum, H., F. Vial, A. Manson, R. Giraldwz, and M. Massebeuf, Nonlinear interaction between the diurnal and semidiurnal tides; terdiurnal and diurnal secondary waves, J. Atmos. Terr. Phys., 51, 627-634, 1989.

Torrence, C., and G. Compo, A practical guide to wavelet analysis, Bull. Am. Meteorol. Soc., 79, 61-78, 1998.

Vial, F., Numerical simulations of atmospheric tides for solstice conditions, J. Geophys. Res., 91, 8955-8969, 1986.

Vial, F., Causes of tidal variability, in, NATO ASI Series Coupling processes in the lower and middle atmosphere, Eds. Thrane E., T. Blix, D. Fritts, Kluwer Academic, 1992.

Vincent, R., Long-period motions in the equatorial mesosphere, J. Atmos. Sol.-Terr. Phys., 55, 1067-1080, 1993.

Vincent, R., T. Tsuda, and S. Kato, Asymmetries in mesospheric tidal structure, J. Atmos. Terr. Phys., 51, 609-616, 1989.

Walterscheid, R., and R. Vincent, Tidal generation of the phased locked 2-day wave in the southern hemisphere summer by wavewave interactions, J. Geophys. Res., 101, 26567-26576, 1996.

Williams, C., and S. Avery, Analysis of long-period waves using the mesosphere-stratosphere-troposphere radar at Poker Flat, Alaska, J. Geophys. Res., 97, 20855-20861, 1992. 